\title{
Corrupção e Crescimento: os impactos da corrupção ponderados pelos diferentes graus de eficiência entre as firmas *
}

Leonardo Andrade Rocha

Professor - Universidade Federal Rural do Semi-Árido (UFERSA) Endereço: Av. Francisco Mota, 572 - Mossoró - Rio Grande do Norte CEP: 59625-900 - E-mail: leonardoandrocha@yahoo.com.br

\author{
Ahmad Saeed Khan \\ Professor - Universidade Federal do Ceará (UFC) \\ Endereço: Rua Campus do Pici - Bloco 826 - Fortaleza/CE \\ CEP: 60455-900 - E-mail: saeed@ufc.br
}

Patrícia Verônica Pinheiro Sales de Lima

Professora - Universidade Federal do Ceará (UFC)

Endereço: Rua Campus do Pici - Bloco 826 - Fortaleza/CE

CEP: 60455-900 - E-mail: patricialima@ufc.br

\section{Maria Ester Soares Dal Poz}

Professora - Universidade Estadual de Campinas (UNICAMP)

Endereço: Rua Pedro Zaccaria, 13083-857 - Limeira/SP

Caixa-postal: 6135 - E-mail: ester.dalpoz@fca.unicamp.br

Recebido: 22/12/2014. Aceite 18/08/2016.

\section{Resumo}

O presente estudo analisou os impactos da corrupção no crescimento das firmas conforme diferentes 'graus' de eficiência. Usando uma técnica não paramétrica de escores de eficiência, foi estimado um modelo de regressão linear interagindo os diferentes escores com um índice de corrupção. Os resultados apontam que a corrupção apresenta impactos negativos nas firmas com baixa performance, ao contrário das firmas com alta performance. Neste sentido, a posição da firma eficiente conforme a fronteira quantílica condiciona significativamente o custo da corrupção, sugerindo um efeito heterogêneo que não é precisamente capturado pelas técnicas tradicionais. Tais resultados corroboram com importantes estudos destacando Batra, Kaufmann e Stone (2003), Wang e You (2012) e Jiang e Nie (2014).

\section{Palavras-Chave}

Eficiência. Corrupção. Desempenho. Crescimento.

\begin{abstract}
This study examined the impact of corruption on growth of firms with different levels of efficiency. The study was made from the non-parametric efficiency scores technique,

- Este artigo é resultado de estudo realizado no âmbito de projeto de pesquisa. OS CUSTOS DA CORRUPÇÃO NA TRAJETÓRIA TECNOLÓGICA: O "câncer" do desenvolvimento econômico, financiado pelo Conselho Nacional de Desenvolvimento Científico e Tecnológico $-\mathrm{CNPq}$.
\end{abstract}


was adjusted a linear regression model interacting the different scores with a corruption index. The results have shown that corruption has negative impacts on low-performing firms, unlike high-performing firms. In this sense, the position of an efficient firm as the quantile frontier affects significantly the consequences of corruption, suggesting a heterogeneous effect that is not accurately captured by traditional techniques. These results corroborate scientific studies carried out by Batra, Kaufmann and Stone (2003), Wang and You (2012) and Jiang and Nie (2014).

\section{JEL Classification}

D61. D73. O3.

\section{Introdução}

A corrupção tem sido um tema de grande debate na literatura econômica, especialmente porque é um importante fator da distorção dos mercados e outras formas de comportamento anticompetitivo, prejudicando todos os agentes envolvidos na atividade econômica. Nenhum país está imune, e a corrupção encontra-se potencialmente presente onde a assimetria de informação é causada por uma restrita rede social, que limita os controles de gestão, além de um sistema judicial e de governança altamente deficiente (ALEXEEV e SONG, 2013).

Em determinados países, como Uganda, aproximadamente 80\% das firmas reportam que precisam pagar algum tipo de propina para permanecerem economicamente ativas. Em Burundi, cerca de 90\% de todas as empresas estabelecidas sugerem que a propina é uma prática padrão na atividade econômica local (SVENSSON, 2003; SCHUMACHER, 2013).

Méndez e Sepúlveda (2006) analisaram os efeitos da corrupção adotando três indicadores de corrupção de importantes organizações de fomento - no crescimento de longo prazo, incorporando métricas de liberdade política como fatores determinantes nesta relação. Os autores identificaram que baixos níveis de corrupção contribuem para o crescimento na tentativa de contornar os abusos da burocracia. Desta maneira, a forma funcional do efeito da corrupção no crescimento se aproximaria de uma forma quadrática, em que a corrupção tem impacto benéfico sobre o crescimento de longo prazo aos baixos níveis de incidência, mas é prejudicial em níveis elevados. 
As conclusões do estudo de Méndez e Sepúlveda (2006) são bastante sugestivas ao serem comparadas com as contribuições de Acemoglu e Verdier (1998). Neste último trabalho, os autores assumem que a existência de corrupção cria a necessidade de fiscalização, a qual gera níveis diferenciados de burocracia. Esses níveis de burocracia algumas vezes podem ser positivos, pois tendem a estimular os agentes privados a implementar melhores projetos de investimento. Assim, se a fiscalização é feita por meio de uma "burocracia adequada", cria-se um sentimento de confiança decorrente de um ambiente institucional favorável ao crescimento de longo prazo. Neste sentido, nota-se que os instrumentos burocráticos de fiscalização estariam associados a uma melhor alocação dos recursos na economia, ${ }^{1}$ e não a níveis elevados de corrupção.

$\mathrm{Na}$ literatura, muitas pesquisas, destacando as contribuições de Shleifer e Vishny (1993), Tanzi (1998), Kaufmann e Wei (1999), Rose-Ackerman (1999) e trabalhos de Treisman (2000), Mocan (2004), Méon e Weill (2010), Dreher e Gassebner (2007), Lambsdorff (2003; 2007) e Banerjee, Mullainathan e Hanna (2012), destacam que os efeitos da corrupção na atividade econômica são claramente "nocivos", embora alguns estudos insistam em uma possível direção contrária.

Essa dicotomia surge a partir de duas importantes hipóteses sob os efeitos da corrupção no crescimento: (1) hipótese da "graxa na engrenagem" e a; (2) hipótese da "areia na engrenagem". Na primeira hipótese, as distorções causadas por instituições ineficientes podem conduzir os agentes a buscarem formas alternativas que acelerem a implementação de seus projetos de investimentos, envolvendo pagamentos aos oficiais públicos na tentativa de contornar os entraves da burocracia. Nesta vertente, encontram-se importantes contribuições de Leff (1964), Bailey (1966), Huntington (1968) e Lui (1985). Ao contrário disso, a segunda hipótese mostra que a corrupção se revela como uma atividade que "supre" o sistema, criando rendas extras aos oficiais corruptos que dependem dela para sobrevivência. Assim, os atrasos ocasionados pelos funcionários corruptos constituem uma forma de oportunidade necessária para extrair subornos a partir desta atividade. Em crítica à hipótese da "graxa", a capacidade dos oficiais públicos em acelerar o pro-

1 ACEMOGLU, D.; ROBINSON, J. Why Nations Fail: The Origins of Power, Prosperity, and Poverty. New York: Crown Publishing Group, 2012. 
cesso pode ser bastante limitada, tendo em vista que a administração consiste em uma sucessão de "centros de decisões". Neste caso, a corrupção não consiste em uma manobra benéfica para a atividade econômica, mas sim uma consequência natural da atividade burocrática. Consequentemente, uma distorção se soma às outras em vez de compensá-las, caracterizando um efeito de "areia na engrenagem". Para uma compreensão detalhada sobre esta literatura, veja os estudos de Meón e Sekkat (2005) e Meón e Weil (2010). A ideia de que a corrupção pode ter efeitos ambíguos sobre o crescimento econômico estimula o debate econômico, especialmente quando se analisam os impactos diretos no desempenho das empresas. ${ }^{2}$

Recentemente, Anokhin e Schulze (2009) analisaram a relação entre o controle da corrupção e o ambiente inovativo das empresas. Os resultados do estudo revelam que um ambiente com alto controle da corrupção está associado a altos níveis de inovação e empreendedorismo. Em ambientes com alta corrupção e excessiva atividade burocrática, os custos de transação, juntamente com outras consequências não produtivas, limitam a escala e o escopo da atividade econômica, reduzindo os níveis de incentivo e aumentando os riscos das atividades voltadas ao empreendedorismo e à inovação. A longo prazo, estas falhas vão somando umas às outras, limitando os esforços em inovação, tanto das firmas locais quanto dos investimentos diretos do exterior.

2 Recentemente, Wang e You (2012) apresentaram evidências empíricas as quais nem todos os países com alta corrupção sofrem com um baixo desempenho no crescimento. Centrando suas análises no crescimento das vendas das firmas chinesas, os autores mostraram que a China, além de outros países, ser considerada um "Paradoxo da Ásia Oriental", tem apresentado um crescimento notavelmente acima de muitas outras economias emergentes, ainda que se observe altos níveis de corrupção. Este "puzzle" é explicado pelas interações entre a corrupção e o efeito do mercado financeiro que concorrem para o crescimento nas vendas das firmas. Esta concorrência entre o efeito financeiro e a corrupção conduz a uma relação de substituição, de forma que um baixo grau de desenvolvimento financeiro pode ser compensado por um aumento da corrupção, estimulando o crescimento nas vendas das firmas. Entretanto, nem sempre este "efeito-substituição" pode promover este crescimento. A explicação consiste nas recentes reformas institucionais que a China vem se adaptando para sustentar o desenvolvimento. Esta transição entre os fatores históricos-institucionais da antiga China para a 'nova' vem acontecendo de maneira mais rápida em alguns setores em relação a outros, forçando as firmas a se adaptarem a esta rápida mudança. Neste caso, a hipótese da "graxa na engrenagem" poderia oferecer fortes subsídios neste fenômeno, tendo em vista que as "propinas" pagas aos oficiais públicos consistem em uma manobra aos entreves burocráticos do sistema em fase de mudança. Além disto, a evidência empírica apresentada pelos autores também se aproxima dos resultados teóricos apresentados por Acemoglu e Verdier (1998). 
Outro importante estudo consiste nas contribuições de Méon e Weil (2010). Os autores analisaram os efeitos da corrupção na produtividade dos países, considerando a influência de outras dimensões de governança nesta relação. Por meio da técnica paramétrica de fronteira estocástica, o termo de ineficiência é afetado pela corrupção e pela burocracia, sendo que esta última, intensifica o efeito da primeira. De acordo com os resultados, a corrupção tem um efeito positivo sobre a eficiência quando a qualidade da governança é muito baixa e, ao contrário, negativa quando a qualidade da governança for alta. As conclusões sugerem que a hipótese da "graxa na engrenagem" é sustentada nos países onde a qualidade da governança é precária, implicando em uma relação negativa entre corrupção e ineficiência. Embora tais resultados apontem um conflito no entendimento sobre as consequências da corrupção e outras "falhas institucionais", poucos estudos, destacando Hallward-Driemeier, Wallstern e Xu (2004), ${ }^{3}$ Batra, Kaufmann e Stone (2003), Gaviria (2002), Smarzynska \& Wei (2002), Asiedu e Freeman (2009) ${ }^{4}$ e Wang e You (2012), analisaram os efeitos da corrupção a em nível de microdados em firmas. Em nenhum destes estudos foram analisadas as influências da corrupção a partir da eficiência observada das firmas.

A presente pesquisa acredita que as duas hipóteses elucidadas sejam fortemente correlacionadas com as diferenças em eficiência ou na distância com a fronteira, cujas firmas se encontram. Isto porque as firmas mais afastadas apresentam importantes limitações, tanto em recursos quanto na formação das suas trajetórias tecnológicas, diferenciando o 'peso' da percepção da corrupção em relação às firmas da fronteira. Anokhin e Schulze (2009) e Acemoglu, Aghion e Zilibotti (2006) apresentaram evidências teóricas que sustentam esta relação.

3 "Such analyses are useful in that they can tell us what factors affect aggregated macro indicators on average. However, economies are heterogeneous and such aggregated analyses cannot tell us which factors may be important within different countries. Firm level data allows us to assess factors that comprise the investment climate on firms themselves." (HALLWARDDRIEMEIER, WALLSTERN, \& XU, 2004, p. 02)

4 "The paucity of research on how corruption affects firm behavior is noted in Svensson (2003, p. 209) who writes that "despite more than two decades of research, however, economic studies on corruption at the firm level are rather limited." Wei (2001, p. 11) conducts an extensive review of the corruption-investment literature and concludes that "firm-level studies are generally rare, for the obvious reason that firm-level data are more difficult to assemble."(ASIEDU \& FREEMAN, 2009, p. 200). 
Contudo, medir a fronteira e, consequentemente, a distância das firmas, não consiste em simples tarefa. As técnicas usuais de regressão linear deixam de capturar características importantes de uma produção tecnologicamente eficiente. A estrutura da produção na fronteira pode diferenciar-se de uma estrutura média de produção construída por uma amostra de dados. A 'melhor prática' não implica necessariamente uma 'prática média', uma vez que ela não incorpora aspectos relativos às economias de escala e escopo ${ }^{5}$ (DARAIO \& SIMAR, 2007).

Assim, buscou-se neste artigo medir o impacto da corrupção no desempenho das empresas, a partir da construção de escores de eficiência por meio da técnica não paramétrica mais recente: fronteiras parciais de ordem- $\alpha$. Esta nova técnica é mais robusta quanto à presença de 'outliers' e incorpora o conceito de quantil para estimar diferentes fronteiras que capturam um padrão de eficiência em relação a $\alpha \%$ da amostra e probabilidade de $(1-\alpha) \%$ de ser pareada com firmas mais eficientes. Neste caso, as firmas eficientes nas fronteiras parciais apresentam uma 'dominância' relativa na amostra que tornase maior na medida em que o quantil converge para a fronteira final $(\alpha=100 \%) .{ }^{6}$ Para cada fronteira estimada, o escore de eficiência é interagido com um índice de corrupção.

Nesse contexto o modelo gera diferentes escores que representam percepções distintas sobre o impacto da corrupção no crescimento das vendas das empresas. Para as firmas ineficientes, ou mais afastadas da fronteira de eficiência, o impacto da corrupção é relativamente maior em comparação com as firmas mais eficientes. Nessa região a corrupção tem um impacto negativo no crescimento. Este aspecto sugere que a corrupção afeta o crescimento das firmas ineficientes como uma "areia na engrenagem". Para as firmas eficientes, as estratégias necessárias para contornar a corrupção somadas às possíveis restrições de recursos podem contribuir para uma assimetria entre

5 "Best practices (captured by the frontier approach) may be better than average practices (measured in a regression-based framework) in the sense that they exploit available substitution possibilities or scale opportunities that average practices do not. Public policy based on the structure of best practice frontiers may be very different from policy based on the structure of average practice functions." (DARAIO \& SIMAR, 2007, p. 02).

${ }^{6}$ O termo de 'dominância' empregado reflete que a fronteira torna-se eficiente para uma parcela da amostra, definida pelo quantil. Assim, firmas eficientes nas fronteiras parciais são ditas 'eficientes' somente comparando com $\alpha \%$ da amostra. Desta forma, tais firmas se configuram como 'dominantes' sob $\alpha \%$ da amostra e probabilidade de $(1-\alpha) \%$ se serem pareadas com firmas mais eficientes. 
as firmas favorecendo especialmente as firmas com maiores taxas de crescimento. Este padrão, mais aderente ao efeito da "graxa na engrenagem", pode explicar a razão das firmas com maiores taxas de crescimento apresentarem padrões superiores com a corrupção em comparação com as firmas com menores taxas.

Tais conclusões permitem supor que nos setores com menores taxas de crescimento entre as firmas, os custos da corrupção são maiores, podendo inviabilizar a construção da sua trajetória. $\mathrm{Na}$ ausência de mecanismos de penalidade, a instituição pode estimular uma assimetria entre as empresas aumentando ainda mais os custos a longo prazo. Esta configuração sugere que nas economias em desenvolvimento, as restrições de crescimento das firmas podem constituir importantes obstáculos que, somados às falhas institucionais, limitam ainda mais a convergência aos padrões da fronteira. Desta forma, uma presença relativamente maior de empresas com baixa performance ou mesmo eficientes com maior grau de dominância podem sugerir padrões mais próximos à "areia na engrenagem" nas economias não desenvolvidas. Nessas economias, em comparação com as economias desenvolvidas, supõe-se maior presença de corrupção estrutural e maior número de firmas mais ineficientes, o que pode representar um custo maior e restrição ao desenvolvimento.

\section{Modelagem empírica}

\subsection{Definição da amostra e operacionalização das variáveis}

Para medir a influência dos esforços inovativos no desempenho das firmas adotou-se a base de dados do The 2013 EU Industrial R\&D Investment Scoreboard (COMMISSION, 2013). Este relatório é publicado anualmente, fornecendo o "ranking" das empresas com o maior volume de gastos em Pesquisa \& Desenvolvimento (P\&D), distribuídas em 40 setores e 46 países no mundo. As informações incluem, além desta variável, o volume das vendas ( $€$ milhões), número de empregados, despesas com capital ( $€$ milhões), rentabilidade, além das medidas de crescimento (para maiores detalhes ver Apêndice A). 
A cada ano, o "ranking" das empresas altera, conferindo perdas de informações sobre algumas empresas em diferentes pontos do tempo. Com o intuito de maximizar o tamanho da amostra para 2.000 empresas (no relatório mais recente de 2013), optou-se por uma base de dados exclusiva para este ano. Sem muitas perdas, acredita-se que uma amostra de 2.000 firmas seja significativa para as conclusões do estudo.

\subsection{Estimando a fronteira}

$\mathrm{Na}$ teoria econômica tradicional é comum presumir que as empresas atuam otimizando suas operações por meio do não desperdício de recursos. Resumidamente, assume-se que os produtores sempre operam em algum lugar na fronteira de seus conjuntos de possibilidade de produção. Contudo, as evidências empíricas sugerem uma realidade bastante diferente: nem todas as firmas são bem sucedidas em diversas circunstâncias, de maneira que algumas podem atuar na fronteira ou sua vizinhança e outras mais afastadas. Neste sentido, é importante para a análise de eficiência investigar o grau com que as firmas não conseguem otimizar suas operações e o quanto se distanciam relativamente de um padrão de referência (fronteira).

$\mathrm{Na}$ teoria econômica a análise de eficiência ganhou bastante notoriedade desde as contribuições de Koopmans (1951), Debreu (1951), e Farrell (1957). Dois importantes métodos ganham destaque nesta abordagem: métodos paramétricos e não paramétricos.

Na primeira técnica, a fronteira de produção é definida por uma função que é matematicamente conhecida e dependente de k-parâmetros desconhecidos, mas que podem ser estimados por meio de técnicas de regressão linear (análise de fronteira estocástica). Além da especificação sobre a fronteira de produção, a facilidade na interpretação econômica dos parâmetros além das propriedades estatísticas que permitam uma análise inferencial, constituem as principais vantagens desta técnica. Apesar de tais vantagens, a técnica não está isenta de limitações. Neste caso, a forma funcional da fronteira a ser estimada, a restrição no uso de múltiplos produtos, além dos pressupostos no 'Processo Gerador dos Dados' (PDG), tornam esta técnica menos atraente em relação aos métodos não paramétricos (BADIN 
\& DARAIO, 2012; SCHWARZ, VAN BELLEGEM, \& FLORENS, 2012; WILSON, 2012; SIMAR \& WILSON, 2013). ${ }^{7}$

Como abordagem alternativa, os métodos não paramétricos (DEA/ $\mathrm{FDH})^{8}$ não dependem de pressupostos no PDG que regem o conjunto de produção das firmas, além de formas funcionais na especificação da fronteira, uma vez que este procedimento emprega o comparativo entre as observações para se definir a referência na amostra (DARAIO \& SIMAR, 2007). Contudo, a natureza determinística e a dificuldade de inferência estatística consistem nas principais limitações desta técnica. Estudos vêm abordando o problema da inferência e do "determinismo", destacando técnicas por meio de resultados assintóticos, conforme Simar e Wilson (2000a), máxima verossimilhança de acordo com Kumbhakar et al. (2007) e métodos de bootstrap segundo Simar e Wilson (1998; 2000b) e Kneip, Simar e Wilson (2003).

Por outro lado, ambas abordagens estão sempre sujeitas a dois problemas comuns nas séries econômicas: (i) pontos discrepantes na amostra ("outliers") e (ii) "maldição da dimensionalidade". ${ }^{9}$ No primeiro caso, a presença de valores extremos desloca a fronteira estimada, levando os escores de eficiência a um claro viés. Desta forma, em qualquer das técnicas, percebe-se uma sensibilidade dos escores

7 Além disto, estes métodos não apresentam robustez quando a forma da distribuição assumida não se sustenta. Neste caso, os outliers presentes nos dados afetam a forma funcional da fronteira, viesando a estrutura da perturbação estocástica que, na maioria das vezes, não está corretamente especificada. Juntando a isto, problemas associados à heterocedasticidade, quando não são propriamente corrigidos, repercute em um viés significativo na definição da fronteira. Em direção contrária, os modelos não paramétricos tornam-se mais atraentes, uma vez que contam com poucas hipóteses no PDG, entretanto, acabam sendo bastante sensíveis à presença de valores extremos e à "maldição da dimensionalidade" (FLORENS, SIMAR, \& KEILEGOM, 2014; BADIN, DARAIO, \& SIMAR, 2014).

8 Na primeira técnica (DEA - Data Envelopment Analysis), o conjunto de produção das firmas $\Psi$ assume os pressupostos de livre disponibilidade (ou possibilidade de desperdício nos recursos) e convexidade (CHARNES, COOPER, \& RHODES, 1978). O conjunto $\mathcal{P}_{D E A}=\left\{(x, y) \in \mathbb{R}_{+}^{p+q} \mid y \leq \sum_{i=1}^{n} \gamma_{i} Y_{i} ; x \geq \sum_{i=1}^{n} \gamma_{i} X_{i} \therefore \sum_{i=1}^{n} \gamma_{i}=1 ; \forall \gamma_{i} \geq 0\right\}$ pode ser reformulado conforme 3 possibilidades: retornos constantes de escala $\left(\sum_{i=1}^{n} \gamma_{i}=1\right)$ é descartado na solução), retornos decrescentes de escala $\left(\sum_{i=1}^{n} \gamma_{i} \leq 1\right)$ e retornos crescentes de escala $\left(\sum_{i=1}^{n} \gamma_{i} \geq 1\right)$. Na segunda abordagem (FDH - Free Disposal Hull) o pressuposto de convexidade é relaxado, tendo em vista que problemas associados às economias de escala, especialização nos fatores de produção, indivisibilidade nos insumos e produtos, imperfeições de mercado, podem não sustentar o emprego deste pressuposto $-\mathcal{P}_{F D H}=\left\{(x, y) \in \mathbb{R}_{+}^{p+q} \mid y \leq \sum_{i=1}^{n} Y_{i} ; x \geq \sum_{i=1}^{n} X_{i} \therefore \forall\left(X_{i}, Y_{i}\right) \in \mathcal{S}_{n}\right\}$ onde $S_{n}=\left\{\left(X_{i}, Y_{i}\right): i=1,2,3 \ldots, n\right\}$ é um conjunto amostral. Assim, o estimador FDH acaba sendo mais amplo em comparação ao tradicional DEA (DEPRINS, SIMAR, \& TULKENS, 1984).

9 "The curse of dimensionality, shared by many nonparametric methods, means that to avoid large variances and wide confidence interval estimates a large quantity of data is needed." (DARAIO \& SIMAR, 2007, p. 26) 
calculados a partir da presença de tais anomalias. No segundo caso, a precisão estatística nos intervalos de confiança depende de um número considerável de observações. Nas técnicas não paramétricas tradicionais (DEA/FDH), a taxa de convergência depende inversamente da dimensão do espaço insumo-produto, de forma que quanto maior o número de p-insumos e q-produtos, maior o tamanho da amostra necessário para amenizar a imprecisão estatística dos escores de eficiência (DARAIO \& SIMAR, 2007; SIMAR \& WILSON, 2013).

Diante destas limitações, técnicas não paramétricas recentes vêm ganhando notoriedade no debate acadêmico por se tornarem mais robustas quanto às restrições mencionadas. Estas técnicas são denominadas de abordagens de 'fronteiras parciais' e consistem no mais moderno campo de estudo em análise de fronteira. Este tema baseia-se principalmente nas contribuições de Cazals, Florens e Simar (2002), Daouia and Simar (2004), Aragon, Daouia e Thomas-Agnan (2005) e Daraio e Simar (2005).

\subsubsection{Abordagens baseadas em 'Fronteiras Parciais'}

O maior benefício desta abordagem consiste em superar as principais desvantagens nas técnicas tradicionais não paramétricas: inferência estatística, dimensionalidade e outliers. Duas categorias para estimação de fronteiras parciais se destacam: (1) fronteiras robustas de ordem-m e; (2) fronteiras robustas de ordem- $\alpha .^{10}$

Na primeira categoria, um número inteiro de m-firmas é aleatoriamente sorteado na amostra para uma comparação com uma unidade tomadora de decisão (DMU). Resumidamente, Cazals, Florens e Simar (2002) introduziram o conceito de "fronteira de produção máxima esperada" de ordem $m \in \mathbb{N}^{*}$, onde $\mathbb{N}^{*}$ corresponde ao conjunto de todos os números inteiros $m \geq 1$. Este conceito é definido como o nível de produção esperada máxima a ser alcançada entre as $m$-firmas sorteadas na população usando menos recursos que a unidade comparada. Assim, a fronteira é definida por cada m-grupo de firmas fixado, de forma que as $m$-fronteiras estimadas convergem para a fronteira total na medida em que $\mathrm{m} \rightarrow \infty .{ }^{11}$ Devido à natureza de corte na fronteira

${ }^{10}$ Os estimadores DEA e FDH envolvem completamente todas as observações da amostra sendo, por esta razão, muitas vezes denominados de estimadores de envoltória completa. Para maiores detalhes, ver Simar \& Wilson (2013). 
de ordem-m, o estimador não cobre todas as observações, de forma que o estimador se torna mais robusto que o tradicional FDH. Os diferentes cortes da amostra geram diferentes fronteiras que repercutem em escores de eficiência menos sensíveis à presença dos valores extremos, que são tratados com DMU's supereficientes ao invés da própria fronteira.

Contudo, uma abordagem alternativa proposta por Aragon, Daouia e Thomas-Agnan (2005) demonstra que o conceito de quantis condicionais se adequa de forma mais robusta em comparação ao método de ordem-m. Nesta abordagem alternativa, o corte amostral é tratado de forma contínua $\alpha \in[0,1]$, de maneira que o conceito dos quantis abrange recortes proporcionais da amostra que podem ser interpretados como probabilidade. O método dos quantis é tradicionalmente conhecido por ser mais robusto quanto à presença de outliers (KOENKER \& HALLOCK, 2001; KOENKER, 2005). Assim, a ideia por trás da fronteira de ordem- $\alpha$ consiste em determinar primeiro a probabilidade $(1-\alpha)$ dos pontos observados situarem acima da fronteira- $\alpha$ estabelecida. Usando a formulação probabilística, as fronteiras de ordem-m podem ser facilmente adaptadas para uma nova formulação baseadas em quantis de ordem- $\alpha$, garantindo ganhos de robustez.

\subsubsection{Fronteiras parciais de ordem- $\alpha$}

O PDG pode ser descrito em termos que permita uma interpretação probabilística acerca dos escores de eficiência, providenciando uma nova forma de interpretação sob a natureza dos estimadores não paramétricos. Assim, para compreender o método de fronteira parcial por quantil, precisamos definir importantes conceitos de função de distribuição de probabilidade.

* DEFINIÇÃO 1: Seja o conjunto amostral $S_{n}=\left\{\left(X_{i}, Y_{i}\right): i=1,2, \ldots, n\right\}$ de niveis de insumo-produto factíveis para uma atividade de interesse, a probabilidade de uma DMU operando nos níveis de insumo-produto $(\boldsymbol{x}, \boldsymbol{y})$ seja dominada é dado por $H_{X Y}(\boldsymbol{x}, \boldsymbol{y})=\operatorname{Pr}(\boldsymbol{X} \leq \boldsymbol{x}, \boldsymbol{Y} \geq \boldsymbol{y})$.

${ }^{11}$ Quando $\mathrm{m} \rightarrow \infty$ o estimador de fronteira parcial de ordem-m converge para o estimador FDH. 
Conforme a Definição $1, H_{X Y}(\boldsymbol{x}, \boldsymbol{y})$ mostra a probabilidade de qualquer outra unidade produzindo no mínimo o nível de produto $\mathrm{y}$, enquanto usa não mais insumos ao nível x para produzir. No caso do estimador tradicional FDH, a fronteira total garante uma probabilidade igual a zero para as unidades operantes na fronteira, uma vez que o procedimento de estimação cobre todos os dados da amostra e nenhuma observação é prevista além da fronteira.

No exemplo produto-orientado, os escores de eficiciência podem ser definidos para a fronteira total:

FDH: $\tilde{\lambda}\left(x, y \mid H_{X Y}(\boldsymbol{x}, \boldsymbol{y})\right)=\sup \{\lambda \mid \operatorname{Pr}(\boldsymbol{X} \leq \boldsymbol{x}, \boldsymbol{Y} \geq \boldsymbol{y})>0\}$

$$
\tilde{\lambda}\left(x, y \mid H_{X Y}(\boldsymbol{x}, \boldsymbol{y})\right)=\sup \left\{\lambda \mid H_{X Y}(\boldsymbol{x}, \lambda \boldsymbol{y})>0\right\}
$$

O escore de eficiência pode ser interpretado como um aumento proporcional no produto necessário para a unidade $(\boldsymbol{x}, \boldsymbol{y}) \in \mathcal{S}_{n}$ alcançar uma probabilidade igual a zero de ser inicialmente dominada por outra DMU escolhida aleatoriamente. Na medida que que a produção sofre o aumento proporcional a garantir uma probabilidade nula de ser dominada, dizemos que a unidade produtiva alcançou a fronteira total FDH. Esta reformulação probabilística apresenta uma forma alternativa para os escores de eficiência tradicionais de DebreuFarrel. Se o conjunto de produção $(\mathcal{P})$ admite o pressuposto de livre disponibilidade (Free Disposal), $\log \mathcal{S}_{n} \subset \mathcal{P}$, isto implica em: $\tilde{\lambda}\left(x, y \mid H_{X Y}(\boldsymbol{x}, \boldsymbol{y})\right)=\tilde{\lambda}(x, y \mid \mathcal{P})$. Neste caso o estimador FDH, $\mathcal{P}_{F D H}=\left\{(x, y) \in \mathbb{R}_{+}^{p+q} \mid y \leq \sum_{i=1}^{n} Y_{i} ; x \geq \sum_{i=1}^{n} X_{i} \therefore \forall\left(X_{i}, Y_{i}\right) \in \mathcal{S}_{n}\right\}$, pode ser reformulado conforme apresentado no parágrafo anterior.

Para se determinar a fronteira do conjunto de produção produto-orientado, precisamos decompor $H_{X Y}(\boldsymbol{x}, \boldsymbol{y})$ utilizando o Teorema de Bayes:

$$
H_{X Y}(\boldsymbol{x}, \boldsymbol{y})=\underbrace{\operatorname{Pr}(Y \geq y \mid X \leq x)}_{S_{Y \mid X}(y \mid x)} \cdot \underbrace{\operatorname{Pr}(X \leq x)}_{F_{X}(x)}=\underbrace{\operatorname{Pr}(X \leq x \mid Y \geq y)}_{F_{X \mid Y}(x \mid y)} \cdot \underbrace{\operatorname{Pr}(Y \geq y)}_{S_{Y}(y)}
$$

Logo, a função-fronteira da produção, $y^{\partial}(x)=\psi(x) \therefore \psi(x): \mathbb{R}_{+}^{p} \rightarrow \mathbb{R}_{+}^{q}$, reflete o nível de produção máxima que pode ser alcançada a partir de um dado nível de insumos. Assim, a função-fronteira corresponde a uma transformação dos insumos em produtos 
para uma firma que possua probabilidade nula de ser dominada: $\psi(x)=\sup \left\{y \mid S_{Y \mid X}(y \mid x)>0, \forall x \in \mathbb{R}_{+}^{p}\right\}$

Graficamente a função $\psi(x)$ pode ser vista da seguinte forma (Figura 1):

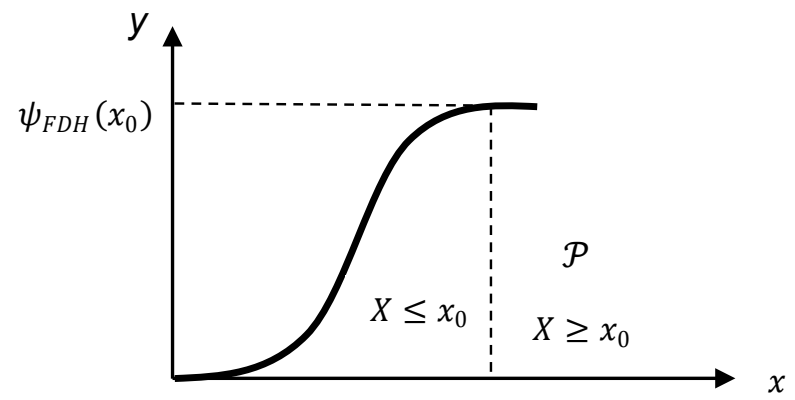

Figura 1- Fronteira produto-orientada para $\mathrm{p}=\mathrm{q}=1$.

No caso de um estimador de fronteira parcial de ordem- $\alpha$, o pareamento das firmas com relação a uma única fronteira total é substituído por um quantil situando-se bem próximo da fronteira, de forma que as DMU's que operam na fronteira têm probabilidade de (1- $\alpha$ ) de serem dominadas por outras firmas com produção igual ou superior (Figura 2).

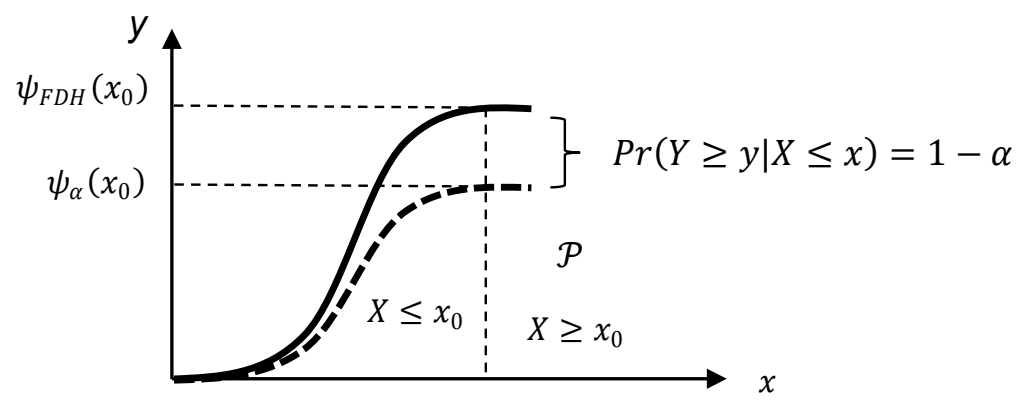

Figura 2- Fronteira produto-orientada para o quantil $\alpha \%$.

Conforme a Figura 2, pode-se apresentar a definição da fronteira eficiente ao nível de $\alpha \%$. Para um dado vetor de insumo-produto $(\mathbf{x}, \mathbf{y})$, o nível de produção eficiente de ordem $\alpha \%$ é dado por: $y_{\alpha}^{\partial}(x)=\tilde{\lambda}_{\alpha}(x, y) \cdot y \equiv \psi_{\alpha}(x)$. Por construção, uma DMU operando no ponto $\left(x, y_{\alpha}^{\partial}(x)\right) \in \mathcal{P}$ tem probabilidade $H_{X Y}\left(\boldsymbol{x}, y_{\alpha}^{\partial}(x)\right)=(1-\alpha) \cdot F_{X}(x)$ de ser dominada. Logo, percebe- 
se que $(1-\alpha)=S_{Y \mid X}(y \mid x) \therefore \alpha=1-S_{Y \mid X}(y \mid x)$ ロ. Assim, o escore de eficiência produto-orientado pode ser apresentado conforma a definiç̃̃o:

* DEFINIÇÃO 2: Para todo x tal que $F_{X}(x)>0$ e $\alpha \in(0,1]$ o escore de eficiência produto-orientado para uma DMU operando em $(x, y) \in \mathcal{P}$ é definido como: $\tilde{\lambda}_{\alpha}(x, y)=\sup \left\{\lambda \mid S_{Y \mid X}(\lambda y \mid x)>1-\alpha\right\}$

A partir da definição, na medida em que o quantil converge monotonicamente $\alpha \rightarrow 1$, a fronteira parcial $\alpha \%$ converge para a fronteira total FDH, logo: $\lim _{\alpha \rightarrow 1} \ddot{\lambda}_{\alpha}(x, y)=\grave{\lambda}(x, y \mid \mathcal{P})$.

Uma vez definido o quantil ou os diferentes quantis, os escores de eficiência para as DMU's podem assumir valores que satisfazem $\tilde{\lambda}_{\alpha}(x, y) \geq 0$. Ao contrário dos escores tradicionais $\tilde{\lambda}_{F D H}(x, y) \geq 1$, os valores estimados permitem a retração no produto para abranger as unidades que agora são supereficientes. $\mathrm{Na}$ interpretação tradicional, o escore calculado aumenta a produção das DMU's ineficientes de forma a deslocarem para um nível de produção eficiente, a partir de uma dada combinação de insumos. No caso da abordagem quantílica, as firmas supereficientes correspondem a uma probabilidade $(1-\alpha) \%$ de dominarem as firmas que operam na fronteira $\alpha \%$. Neste caso, a produção precisa ser reduzida para operarem em um nível balanceado de eficiência para a fronteira parcial de ordem- $\alpha$.

\subsection{Metodologia econométrica}

Para analisar os impactos da corrupção, em diferentes países, nas vendas das firmas foi estimada a seguinte equação:

$$
\begin{gathered}
g_{i j c}=\beta_{0}+\beta_{1} \cdot C C_{c}+\beta_{2} \cdot \ln (R \& D)_{i j c}+\quad \text { ME. } 1 \\
\beta_{3} \cdot \ln (L)_{i j c}+\beta_{4} \cdot \tilde{\lambda}_{\alpha}(x, y)_{i j c} \cdot C C_{c}+\mu_{j}+\delta_{c}+\varepsilon_{i j c}
\end{gathered}
$$

Conforme a equação ME.1 as variáveis representam, respectivamente, a taxa de crescimento das vendas nos últimos 3 anos, ${ }^{12}$ ao índice de corrupção de cada país, aos investimentos em P\&D e ao número de funcionários da firma "i", no setor "j" e no país "c". Com relação ao indicador de corrupção, este foi extraído do The

$\overline{12}$ Taxa de crescimento anual composta ou 'Compound Annual Growth Rate' (CAGR). 
Worldwide Governance Indicators (WGI) compilado pelo Banco Mundial (KAUFMANN, KRAAY, \& MASTRUZZI, The Worldwide Governance Indicators: Methodology and Analytical Issues, 2013). $\mathrm{O}$ indicador calculado foi normalizado no intervalo $C C_{c} \in(0,1)$, de forma que quanto maior o valor (mais próximo de 1 ) maior a percepção com que o poder público é exercido para expropriação de ganhos privados, consequentemente as instituições são percebidas mais ineficientes. ${ }^{13}$ Quanto ao uso das vendas como variável dependente ressalta-se que se trata de uma escolha cada vez mais comum na literatura internacional (BOGLIACINO, 2014; BOGLIACINO e CORDONA, 2010; GARCNA-MANJEN e ROMERO-MERINO, 2012; MONTRESOR e VEZZANI, 2015; LIVANIS e LAMIN, 2016).

Com relação ao parâmetro de eficiência, $\tilde{\lambda}_{\alpha}(x, y)_{i j c}$, este na verdade reflete a distância relativa de cada firma em relação à fronteira, dada a combinação de insumos necessários para se obter o máximo de produto. A Tabela 1 resume a combinação de insumo produto na estimação dos escores de eficiência.

Tabela 1 - Definição das variáveis para o cálculo dos escores de eficiência.

\begin{tabular}{|c|c|c|}
\hline Variáveis & Definição da & \\
\hline Y & Vendas totais & \\
\hline $\mathrm{L}$ & Número de empregados & \\
\hline \multirow[t]{5}{*}{ K } & Capex: investimento em bens de capital & \\
\hline & Divisão das variáveis pa & scores \\
\hline & Output & Input \\
\hline & Y & $L$ \\
\hline & - & K \\
\hline
\end{tabular}

Fonte: Elaboração própria.

Com base na definição das variáveis na Tabela 1 , foram calculadas 1 fronteira por setor, totalizando 40 fronteiras. Para cada grupo de setor, foram divididos 5 quantis $(25 \%, 50 \%, 75 \%, 95 \%$ e $100 \%$, sendo este último a fronteira final FDH) para cada quantil foi estimada uma fronteira representativa. Dessa forma, o número total de fron-

\footnotetext{
13 "Control of Corruption (CC) - capturing perceptions of the extent to which public power is exercised for private gain, including both petty and grand forms of corruption, as well as "capture" of the state by elites and private interests." (KAUFMANN, KRAAY, \& MASTRUZZI, The Worldwide Governance Indicators: Methodology and Analytical Issues, 2013, p. 04)
} 
teiras estimadas consiste em $5 \times 40=200$ fronteiras de eficiência na amostra.

Em adição ao modelo $\mu_{j}, \delta_{c}$, representam os conjuntos de efeitos fixos relativos às características ou fatores de heterogeneidade observável entre os setores e aos países, respectivamente, sendo necessário como controle no processo de estimação. Por último, tem-se o termo do erro estocástico, $\varepsilon_{i j c}$, que corresponde a todos os demais fatores comuns, mas que estão de fora do controle estatístico, satisfazendo a condição: $\varepsilon_{i j c} \sim N\left(0, \sigma_{\varepsilon}^{2}\right)$ para todo i,j,c.

O impacto da percepção da corrupção nas vendas das firmas depende do efeito-eficiência que é capturado pelo escore estimado pela fronteira parcial de ordem- $\alpha$. Esta relação pode ser facilmente apresentada pela derivada parcial na função estimada:

$$
\frac{\partial g_{i j c}}{\partial C C_{c}}=\hat{\beta}_{1}+\hat{\beta}_{4} \cdot \tilde{\lambda}_{\alpha}(x, y)_{i j c}
$$

Segundo a equação ME.2, o impacto da corrupção é diferenciado entre as firmas e é capturado pelo coeficiente de elasticidade. Para uma melhor compreensão desta relação, três cenários são dados uma maior ênfase $\left(\tilde{\lambda}_{\alpha}(x, y)_{i j c} \geq 0\right):(i) \lim \tilde{\lambda}_{\alpha}(x, y)_{i j c} \rightarrow 1$, (ii) $\lim \tilde{\lambda}_{\alpha}(x, y)_{i j c} \rightarrow 0$ e (iii) $\lim \tilde{\lambda}_{\alpha}(x, y)_{i i c} \rightarrow \lambda^{*}>1$.

No primeiro caso, estamos aprofundando a análise para o grupo de firmas que se encontram na vizinhança da fronteira. No segundo, estamos abordando o grupo de firmas que operam além da fronteira, neste caso, as firmas supereficientes. Por último, tem-se o grupo de firmas mais afastadas da fronteira e que operam com relativa ineficiência. Em cada caso, diferentes valores no escore de eficiência geram "pesos" diferentes na percepção da corrupção, impactando diretamente nas vendas das empresas.

\subsection{Eficiência das estimativas}

$\mathrm{Na}$ existência de erros não normais e presença de pontos discrepantes na amostra ("outliers"), o método tradicional de mínimos quadrados ordinários é ineficiente, embora consistente, caso o pressuposto de ortogonalidade dos erros não seja violado. Neste caso, a 
confiabilidade das estimativas fica comprometida, uma vez que os intervalos de confiança apresentam claros 'sinais' de tendenciosidade (KOENKER e HALLOCK, 2001).

Assim, um importante procedimento vem sendo aplicado como forma de correção quando o método apresenta sinais de violação nos pressupostos de normalidade. Este procedimento, graças às contribuições de Efron (1979), emprega um método de reamostragem que calcula diferentes estimativas de parâmetros, procedendo com uma média das B-ésimas estimativas amostrais. Tal procedimento é conhecido como Método de Bootstrap.

A maior vantagem desta metodologia, consiste nas suposições de normalidade dos resíduos que não são requisitadas para o emprego do método, garantindo estimativas consistentes da variância dos parâmetros (ver Greene (2012) e Cameron e Trivedi (2005)). Embora bastante sofisticada seja esta técnica, o número de replicações da amostra tem sido algo de muita controvérsia no debate acadêmico. Por exemplo, Efron e Tibshirani (1993) afirmam que 50 replicações são um número suficiente para se obter uma "boa" estimativa de erro-padrão.

Contudo, Andrews e Buchinsky (2000) sugerem um tamanho maior de replicações para obter estimativas mais confiáveis, adotando um número $B=400$. Na presente pesquisa, será aplicado o procedimento apresentado por Andrews e Buchinsky (2000), uma vez que um número maior de replicações permite uma estimativa mais consistente da média dos parâmetros e, consequentemente, dos intervalos de confiança.

\section{Análise dos resultados}

\subsection{Análise descritiva dos dados}

Nesta seção, foram agrupadas as firmas conforme três definições de grupo de setores: setores com alta-intensidade em P\&D, setores com média-intensidade em P\&D e setores com baixa-intensidade em P\&D. Para esta conceituação foram adotados os critérios no relatório supracitado, seguindo a definição dos setores por grau de intensidade em P \& D (Tabela 2). 
Tabela 2 - Definição dos setores por grau de intensidade em P\&D

\begin{tabular}{|c|c|}
\hline Grupo de setores & Intensidade em P\&D (P\&D/Vendas) \% \\
\hline Alta-intensidade & $>5 \%$ \\
\hline Média-intensidade & {$[1 \%, 5 \%]$} \\
\hline Baixa-intensidade & $<1 \%$ \\
\hline \multicolumn{2}{|r|}{ Definição dos setores } \\
\hline Alta-intensidade & $\begin{array}{l}\text { Farmacêutico e biotecnologia; Equipamentos de saúde e serviços; Hardware e equi- } \\
\text { pamentos de tecnologia; Software e serviços de informática; Aeronáutica e Defesa. }\end{array}$ \\
\hline Média-intensidade & $\begin{array}{l}\text { Eletrônica e equipamentos elétricos; Automóveis e autopeças; Engenharia e máqui- } \\
\text { nas industriais; Produtos químicos; Bens de uso pessoal; Produtos domésticos; } \\
\text { Indústria geral; Serviços de suporte técnico; Produtos alimentícios; Bebidas; Viagens } \\
\text { e lazer; Meios de comunicação; Equipamentos para Ind. de Petróleo; Eletricidade; } \\
\text { Telecomunicações fixas. }\end{array}$ \\
\hline Baixa-intensidade & $\begin{array}{l}\text { Geração de petróleo e gás; Metais industriais; Materiais de construção; Varejo de } \\
\text { alimentos e medicamentos; Transporte; Mineração; Tabaco; Multiutilidades. }\end{array}$ \\
\hline
\end{tabular}

Fonte: Adaptado de The 2013 EU Industrial R\&D Investment Scoreboard, 2013 [p.27].

A Tabela 3 abaixo apresenta a distribuição das firmas segundo a intensidade dos investimentos em pesquisa. Conforme a distribuição, a maior proporção das firmas é de média-intensidade em P\&D $(48,75 \%)$, seguidas de alta-intensidade $(40,65 \%)$ e baixa-intensidade $(10,60 \%)$. Na distribuição acumulada, aproximadamente $89 \%$ das firmas são, ao menos, média-intensivas em P\&D.

Tabela 3 - Estatísticas descritivas por intensidade dos investimentos em P\&D

\begin{tabular}{l|c|c|c}
\hline Grupo de Setor & Freq. Abs. & Freq. Rel. (\%) & Freq. Acum. (\%) \\
\hline Media-intensidade & 975 & 48,75 & 48,75 \\
Alta-intensidade & 813 & 40,65 & 89,40 \\
Baixa-intensidade & 212 & 10,60 & 100,00 \\
\hline Total & $\mathbf{2 . 0 0 0}$ & $\mathbf{1 0 0 , 0 0}$ & - \\
\hline
\end{tabular}

Fonte: Elaboração própria.

A Tabela 4 ilustra a distribuição das firmas conforme os critérios de eficiência, ineficiência e supereficiência, a partir de cada quantil selecionado para estimar as fronteias parciais. A cada quantil, os escores estimados alteram a distribuição entre firmas eficientes, ineficientes e supereficientes. Nos quantis iniciais, a proporção de firmas ineficientes tende a ser menor em comparação com as firmas supereficientes. Este aspecto é decorrente do fato de a fronteira parcial no quantil inferior 'deslocar' a distribuição dos dados seguindo uma assimetria, de forma que as firmas eficientes (fronteira) no quantil- $\alpha$ apresentam $(1-\alpha) \%$ de probabilidade de serem 'prepon- 
deradas ou dominadas' por firmas mais eficientes. Assim, as firmas da fronteira no quantil- $\alpha$ são eficientes em comparação com $\alpha \%$ e ineficientes em relação a (1- $\alpha) \%$ da amostra. Este contexto tornase de grande relevância para a análise, uma vez que esta técnica não permite apenas comparar firmas eficientes com firmas ineficientes, mas também comparar firmas eficientes em diferentes contextos de dominância na amostra. Este destaque tem sido uma grande vantagem da técnica de fronteiras parciais em detrimento das abordagens tradicionais (BADIN, DARAIO e SIMAR, 2014). ${ }^{14}$

Tabela 4 - Distribuição das firmas por quantil

\begin{tabular}{|c|c|c|c|}
\hline \multirow{2}{*}{ Por eficiência } & \multicolumn{3}{|c|}{ Quantil 25\% } \\
\hline & Freq. Abs. & Freq. Rel. (\%) & Freq. Acum. (\%) \\
\hline Firmas eficientes & 95 & 4,75 & 4,75 \\
\hline Firmas ineficientes & 375 & 18,75 & 23,50 \\
\hline \multirow[t]{3}{*}{ Firmas supereficientes } & 1.530 & 76,50 & 100,00 \\
\hline & \multicolumn{3}{|c|}{ Quantil $50 \%$} \\
\hline & Freq. Abs. & Freq. Rel. (\%) & Freq. Acum. (\%) \\
\hline Firmas eficientes & 113 & 5,65 & 5,65 \\
\hline Firmas ineficientes & 415 & 20,75 & 26,40 \\
\hline \multirow[t]{3}{*}{ Firmas supereficientes } & 1.472 & 73,60 & 100,00 \\
\hline & \multicolumn{3}{|c|}{ Quantil $75 \%$} \\
\hline & Freq. Abs. & Freq. Rel. (\%) & Freq. Acum. (\%) \\
\hline Firmas eficientes & 238 & 11,90 & 11,90 \\
\hline Firmas ineficientes & 538 & 26,90 & 38,80 \\
\hline \multirow[t]{3}{*}{ Firmas supereficientes } & 1.224 & 61,20 & 100,00 \\
\hline & \multicolumn{3}{|c|}{ Quantil 95\% } \\
\hline & Freq. Abs. & Freq. Rel. (\%) & Freq. Acum. (\%) \\
\hline Firmas eficientes & 503 & 25,15 & 25,15 \\
\hline Firmas ineficientes & 994 & 49,70 & 74,85 \\
\hline \multirow[t]{3}{*}{ Firmas super-eficientes } & 503 & 25,15 & 100,00 \\
\hline & \multicolumn{3}{|c|}{ Quantil $100 \%$} \\
\hline & Freq. Abs. & Freq. Rel. (\%) & Freq. Acum. (\%) \\
\hline Firmas eficientes & 656 & 32,80 & 32,80 \\
\hline Firmas ineficientes & 1.344 & 67,20 & 100,00 \\
\hline Firmas super-eficientes & - & - & - \\
\hline
\end{tabular}

Fonte: Elaboração própria.

Nota: A definição das firmas segue o critério estabelecido na metodologia, $\tilde{\lambda}_{\alpha}(x, y)_{i j c}=1$ para firmas eficientes, $\tilde{\lambda}_{\alpha}(x, y)_{i j c}<1$ para firmas super-eficientes e $\tilde{\lambda}_{\alpha}(x, y)_{i j c}>1$ para firmas ineficientes.

De acordo com as informações da tabela, um fato bastante intuitivo corresponde à proporção de firmas eficientes que aumenta na medida em que 'deslocamos' do quantil inferior de $25 \%$ para o quantil final de $100 \%$. Neste sentido, a proporção passa de $4,75 \%$ no primei-

${ }^{14}$ No Apêndice B encontram-se as tabelas de contingência que cruza as informações das firmas segundo o grau de eficiência e intensidade do setor. 
ro caso para $32,80 \%$. No tocante às firmas ineficientes, a proporção gradativamente aumenta com o deslocamento do quantil. Neste caso em específico, o aumento do quantil proporciona uma redistribuição das firmas eficientes e supereficientes, conduzindo em um aumento gradual na proporção de firmas ineficientes.

Com relação à mediana (as firmas da fronteira são eficientes em relação a $50 \%$ da amostra e $50 \%$ de probabilidade de serem dominadas por firmas mais eficientes), percebe-se assimetria na distribuição, com firmas supereficientes, proporcionalmente bem superiores em relação às firmas ineficientes $(73,60 \%$ contra 5,65\%). Esta proporção tende a se igualar a partir do quantil $75 \%$ (25,15\% para ambas as firmas) e com um tamanho relativamente maior para firmas eficientes $(49,70 \%)$. Quando as fronteiras parciais convergem para a fronteira final (quantil 100\%), as firmas eficientes apresentam quase $1 / 3$ da amostra contra 2/3 de firmas ineficientes. Neste caso, as firmas eficientes apresentam $0 \%$ de probabilidade de serem 'dominadas' por firmas mais eficientes e, portanto, representam a eficiência final em relação às demais firmas.

A Tabela 5 seguinte expõe as estatísticas descritivas em relação às variáveis do modelo, excluindo apenas os escores de eficiência. O investimento médio em $\mathrm{P} \& \mathrm{D}$ pelas empresas representou aproximadamente 269 milhões de euros, considerando um intervalo mínimo de 22,6 milhões e máximo de 9,5 bilhões de euros. Com relação às vendas, o nível médio observado pelas empresas representou 8,4 bilhões de euros. Ao longo dos percentis selecionados, 25\% das firmas apresentaram vendas de no máximo 583 milhões de euros. Já na mediana, $50 \%$ das firmas apresentaram vendas de no máximo 1,88 bilhões de euros.

Tabela 5 - Estatísticas descritivas das variáveis

\begin{tabular}{|c|c|c|c|c|c|c|c|c|}
\hline \multirow{2}{*}{ Variáveis } & \multirow{2}{*}{$\mathrm{N}$} & \multirow{2}{*}{ Média } & \multirow{2}{*}{ Máx. } & \multirow{2}{*}{ Min } & \multicolumn{4}{|c|}{ Percentil } \\
\hline & & & & & $25 \%$ & $50 \%$ & $75 \%$ & $95 \%$ \\
\hline iv. $P \& D(€$ milhões $)$ & 2.000 & 269,40 & $9.515,00$ & 22,60 & 35,80 & 63,50 & 156,40 & $1.104,00$ \\
\hline Vendas(€ milhões) & 2.000 & $8.495,00$ & $354.000,00$ & 0,00 & 583,10 & $1.884,00$ & $6.151,00$ & $38.371,00$ \\
\hline $\begin{array}{l}\text { Taxa de crescimento } \\
\text { - vendas (\%) }\end{array}$ & 2.000 & 12,90 & $1.377,00$ & $-100,00$ & 2,40 & 8,00 & 16,30 & 43,20 \\
\hline Inv. Capex(€ milhões) & 2.000 & 617,90 & $37.566,00$ & 0,002 & 21,30 & 80,40 & 311,50 & $2.481,00$ \\
\hline № Funcionários & 2.000 & $26.516,00$ & $548.355,00$ & 0,00 & $2.437,00$ & $7.971,00$ & $22.545,00$ & $118.087,00$ \\
\hline Índice de Corrupção & 2.000 & 0,23 & 0,75 & 0,02 & 0,18 & 0,22 & 0,22 & 0,60 \\
\hline
\end{tabular}

Fonte: Elaboração própria. 
A taxa de crescimento das vendas apresentou um valor moderado de $12,9 \%$, comparando isto ao mínimo de retração de $100 \%$ e um crescimento máximo de $1.377 \%$. Tomando a mediana, $50 \%$ das firmas apresentaram um crescimento de no máximo $8 \%$. No último percentil, 95\% das firmas apresentaram um crescimento nas vendas de no máximo 43,2\%. No tocante aos investimentos em bens de capital, a média obtida correspondeu a aproximadamente 26,5 bilhões de euros. Este valor consistiu entre um intervalo mínimo de 0,002 milhões de euros e no máximo de 37,5 bilhões de euros. Ainda considerando tais investimentos, 25\% das firmas apresentaram uma aplicação máxima de 21,3 milhões e 75\% com no máximo 311,5 milhões de euros.

A Tabela 6 exibe os valores das variáveis considerando o desempenho global das firmas da amostra. As empresas da amostra investiram 538,8 bilhões de euros em P\&D e 6,2\% a mais em relação ao ano anterior, 2011. Com relação às vendas, o valor situou-se em torno de 16,85 trilhões de euros, um crescimento de 8,5\% em relação a 2011. Para os investimentos em bens de capital o montante aplicado correspondeu a aproximadamente 1,11 trilhões de euros, representando um crescimento de 9,6\% em relação a 2011. Por fim, o estoque de funcionários pelas empresas correspondeu a 48,5 milhões de empregos envolvidos, representando um crescimento na demanda por trabalho de 1,5\% em comparação a 2011.

Tabela 6 - Desempenho global das 2.000 firmas

\begin{tabular}{|c|c|c|}
\hline & Fator & Amostra (2,000 firmas) \\
\hline \multirow{3}{*}{ Inv. P\&D } & Bilhões de euros & 538,80 \\
\hline & Crescimento (1 ano) \% & 6,20 \\
\hline & Crescimento ( 3 anos $\left.{ }^{*}\right) \%$ & 6,40 \\
\hline \multirow{3}{*}{ Vendas } & Bilhões de euros & $16.845,80$ \\
\hline & Crescimento (1 ano) \% & 4,20 \\
\hline & Crescimento ( 3 anos* $) \%$ & 8,50 \\
\hline \multirow{3}{*}{ Inv. Capex } & Bilhões de euros & $1.109,10$ \\
\hline & Crescimento (1 ano) \% & 9,60 \\
\hline & Crescimento (3 anos* ${ }^{*} \%$ & 9,30 \\
\hline \multirow{3}{*}{ № Func. } & Milhões & 48,47 \\
\hline & Crescimento (1 ano) \% & 1,50 \\
\hline & Crescimento (3 anos*) \% & 3,40 \\
\hline
\end{tabular}

Fonte:

Nota: $\left({ }^{*}\right)$ representa a taxa de crescimento anual composta, ou CAGR (em inglês) que é definida pela média geométrica dos últimos três anos, neste caso ao longo do período 2010-2012. 
A Figura 3 ilustra a distribuição dos investimentos em P\&D conforme os principais grupos econômicos.

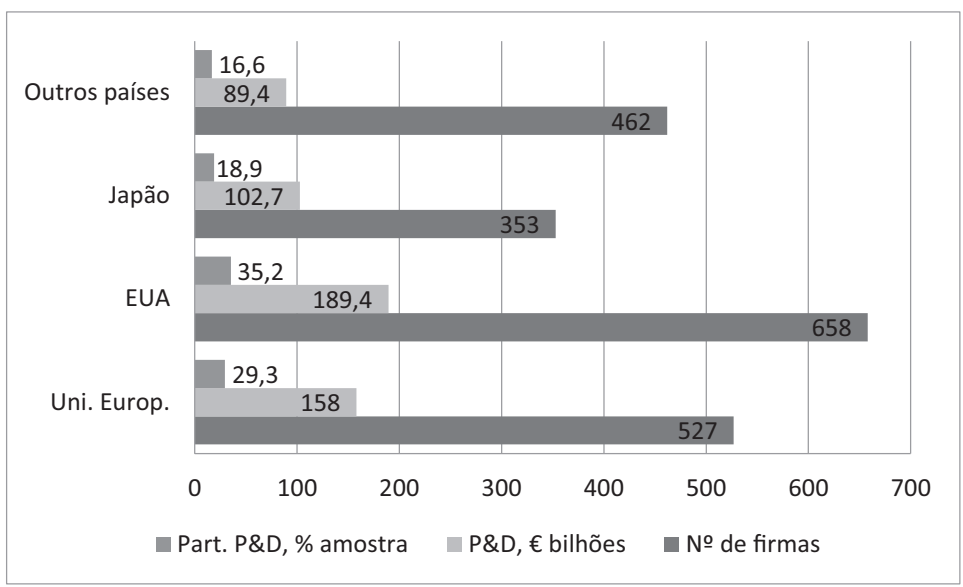

Figura 3 - Distribuição dos investimentos em P\&D por região no mundo. Fonte: Elaboração própria.

A Tabela 7 exibe as estatísticas descritivas dos escores de eficiência por quantil calculado. As 2.000 empresas estão distribuídas em 4 grandes grupos: 527 firmas na União Europeia, 658 firmas nos Estados Unidos, 353 no Japão e 462 firmas em outros países. Em especial neste último grupo, incluem firmas distribuídas em países como China (93), Taiwan (82), Coreia do Sul (56), Suíça (54), Índia (22) além de outros 19 países. O Brasil apresentou um total de 8 firmas na amostra.

A União Europeia respondeu por 29,3\% de todo o investimento em pesquisa aplicado em 2012. Esta parcela equivale a 158 bilhões de euros, representando um crescimento de 6,3\% em relação ao ano anterior (um valor pouco acima do crescimento mundial de $6,2 \%$, conforme dados do relatório). O mercado americano respondeu por um volume de 189,4 bilhões de euros em investimentos em P\&D. Isto representa aproximadamente $20 \%$ a mais do investimento aplicado por empresas da União Europeia. Todo este investimento representa um crescimento de 8,2\% em relação a 2011. Firmas japonesas foram responsáveis por 102,7 bilhões de euros em investimentos em P\&D. Esta quantia representa aproximadamente $54 \%$ do total investido por firmas americanas $(18,9 \%$ do investimento global). Por último, 
os demais países da amostra destinaram 89,4 bilhões em $\mathrm{P} \& \mathrm{D}$, representando $16,6 \%$ do investimento global.

Tabela 7 - Estatísticas descritivas dos escores de eficiência

\begin{tabular}{ccccc}
\hline Variáveis & $\begin{array}{c}(1) \\
\mathbf{N}\end{array}$ & $\begin{array}{c}(2) \\
\text { Média }\end{array}$ & $\begin{array}{c}(3) \\
\text { Máx. }\end{array}$ & $\begin{array}{c}(4) \\
\text { Min. }\end{array}$ \\
\hline & & & & \\
$\lambda \alpha(25 \%)$ & 2.000 & 0,346 & 81,000 & 0,001 \\
$\lambda \alpha(50 \%)$ & 2.000 & 0,608 & 127,500 & 0,001 \\
$\lambda \alpha(75 \%)$ & 2.000 & 1,099 & 287,000 & 0,015 \\
$\lambda \alpha(95 \%)$ & 2.000 & 1,895 & 386,500 & 0,036 \\
$\lambda \alpha(100 \%)$ & 2.000 & 2,612 & 514,500 & 1,000 \\
\hline
\end{tabular}

Fonte: Elaboração própria.

De acordo com os dados apresentados e analisando a média ao longo dos escores parciais, percebe-se um aumento gradativo à medida que deslocamos os quantis em direção à fronteira final. Este aspecto demonstra que o 'peso' das firmas ineficientes aumenta o escore médio na medida em que a fronteira parcial altera a assimetria na distribuição.

O conceito de assimetria está relacionado ao corte que a fronteira exerce em cada quantil. A exemplo, no quantil de $25 \%$ as firmas da fronteira são eficientes sob $25 \%$ da amostra e com $75 \%$ de probabilidade de serem ineficientes com comparação com as firmas 'além' da fronteira. Neste caso, a proporção de firmas supereficientes subestima a média do escore. À proporção que a fronteira parcial descola ao longo dos diferentes quantis em direção à fronteira final, as mudanças na composição da 'dominância' alteram o efeito médio do escore e passam a superestimar o efeito médio do escore de eficiência. Este fato fica mais claro quando se analisa conjuntamente os valores máximos que passam a aumentar com o deslocamento dos quantis em direção à fronteira final.

Comparando os valores máximos, a firma mais ineficiente precisa ajustar suas vendas em 81 milhões de euros no quantil 25\% e passa a ajustar para 514,5 milhões de euros na fronteira final. Esta variação representa aproximadamente um crescimento de 535\%. 
Analisando estas 'distorções' sob o efeito da corrupção, o sentido da relação corrupção x crescimento dependerá da posição da firma em comparação com a fronteira analisada. Firmas ineficientes sob um pareamento com firmas da fronteira e com baixo grau de dominância podem apresentar efeitos distintos quando comparadas com a fronteira final. Isto decorre especialmente da influência de "outliers" que passam a definir a fronteira final 'tendenciando' possivelmente esta relação. Neste caso em específico, a fronteira final pode distorcer a compreensão que a corrupção exerce em diferentes firmas. Assim, firmas supereficientes e que não necessariamente sejam a referência no pareamento (definidas como fronteira) podem apresentar uma relação distinta com a corrupção, mesmo em comparação com as firmas da fronteira. Os resultados do modelo, a seguir, complementam essas reflexões.

\subsection{Resultados do modelo econométrico}

A Tabela 8 apresenta os primeiros resultados da equação estimada ME.1 incluindo somente os efeitos fixos de país. O aumento dos investimentos em $\mathrm{P} \& \mathrm{D}$ estão associados a maiores taxas de crescimento nas vendas das empresas. $\mathrm{O}$ aumento de $1 \%$ nestes investimentos contribui de $1,4 \%$ a $1,6 \%$ no crescimento das vendas (parâmetros significativos ao nível de 1\%). Este efeito positivo apresentou magnitude crescente com os quantis sugerindo que, nos quantis superiores cuja probabilidade de dominância nas firmas eficientes é menos perceptiva, o impacto do investimento no crescimento vai aumentando gradativamente. 
Tabela 8 - Resultados da equação ME.1

\begin{tabular}{|c|c|c|c|c|c|}
\hline \multirow{3}{*}{ VARIÁVEIS } & \multicolumn{5}{|c|}{ Variável dependente: crescimento das vendas (últimos 3 anos) } \\
\hline & (1) & (2) & $(3)$ & (4) & (5) \\
\hline & Quantil 25\% & Quantil $50 \%$ & Quantil 75\% & Quantil 95\% & FDH \\
\hline \multirow[t]{2}{*}{$\ln (\mathrm{R} \& \mathrm{D})$} & $1.438^{\star \star \star}$ & $1.590^{\star \star \star}$ & $1.588^{\star \star \star}$ & $1.628^{\star * *}$ & $1.637^{\star \star *}$ \\
\hline & $(0.528)$ & $(0.525)$ & $(0.524)$ & $(0.525)$ & $(0.525)$ \\
\hline \multirow[t]{2}{*}{$\ln (L)$} & $-4.247^{\star \star *}$ & $-4.412^{\star \star *}$ & $-4.331^{\star * \star}$ & $-4.356^{* * *}$ & $-4.311^{* * *}$ \\
\hline & $(0.954)$ & $(0.950)$ & $(0.944)$ & $(0.943)$ & $(0.939)$ \\
\hline \multirow[t]{2}{*}{ CC } & $114.4^{*}$ & $112.0^{*}$ & $112.2^{*}$ & $112.1^{*}$ & $113.2^{*}$ \\
\hline & $(60.23)$ & $(59.90)$ & $(59.96)$ & $(59.90)$ & (59.91) \\
\hline \multirow[t]{2}{*}{$C C^{\star} \lambda_{25}(\mathrm{x}, \mathrm{y})$} & $-8.967^{\star \star *}$ & & & & \\
\hline & $(2.284)$ & & & & \\
\hline \multirow[t]{2}{*}{$\mathrm{CC}^{\star} \lambda_{50}(\mathrm{x}, \mathrm{y})$} & & $-5.809^{* * *}$ & & & \\
\hline & & $(0.712)$ & & & \\
\hline \multirow[t]{2}{*}{$C C^{\star} \lambda_{75}(x, y)$} & & & $-2.512^{\star \star \star}$ & & \\
\hline & & & $(0.467)$ & & \\
\hline \multirow[t]{2}{*}{$C C^{\star} \lambda_{95}(x, y)$} & & & & $-1.673^{\star \star \star}$ & \\
\hline & & & & $(0.114)$ & \\
\hline \multirow[t]{2}{*}{$\mathrm{CC}^{\star} \lambda_{100}(\mathrm{x}, \mathrm{y})$} & & & & & $-1.368^{\star \star \star}$ \\
\hline & & & & & $(0.174)$ \\
\hline Amostra & 2,000 & 2,000 & 2,000 & 2,000 & 2,000 \\
\hline $\mathrm{R}^{2}$ & 0.056 & 0.060 & 0.059 & 0.060 & 0.059 \\
\hline Teste Wald $\left(\chi^{2}\right)$ & $56.13^{\star \star \star}$ & $95.17^{\star \star \star}$ & $62.68^{\star \star \star}$ & $177.14^{* * *}$ & $136.29^{* * *}$ \\
\hline Teste Heterocedasticidade & $3.37^{\star}$ & $3.22^{*}$ & $3.11^{*}$ & $3.09^{*}$ & $3.00^{*}$ \\
\hline Ef.Fixos-Pais & SIM & SIM & SIM & SIM & SIM \\
\hline Ef.Fixos-Setor & NAO & NAO & NAO & NAO & NAO \\
\hline
\end{tabular}

Fonte: Elaboração própria.

Nota: Os asteriscos representam os respectivos p-valores; ${ }^{* * *} \mathrm{p}<0.01,{ }^{* *} \mathrm{p}<0.05,{ }^{*} \mathrm{p}<0.1$. As estimativas de erro-padrão foram corrigidas a partir da técnica de bootstrap com 400 réplicas.

Com relação ao número de funcionários, o parâmetro estimado apresentou um sinal negativo em todos os quantis, oscilando entre $-4,2 \%$ a $-4,4 \%$ na taxa de crescimento (parâmetros significativos ao nível de $1 \%)$. Este padrão indica que nas empresas intensivas em mão de obra, o aumento deste recurso tende a comprometer a taxa de crescimento, ao contrário dos investimentos em P\&D. 
Para avaliar o efeito final da corrupção no crescimento das vendas, aplica-se a derivada parcial da taxa de crescimento com relação ao indicador de corrupção ao longo dos quantis (ME.2): $\frac{\partial g_{i j c}}{\partial C C_{c}}=\hat{\beta}_{1}-\hat{\beta}_{4} \cdot \tilde{\lambda}_{\alpha}(x, y)_{i j c}$. Tomando como exemplo as firmas eficientes, o efeito final da corrupção é positivo no crescimento. Este efeito final gradativamente aumenta quando deslocamos a fronteira para os quantis superiores, abrangendo as firmas com menores graus de dominância na amostra.

Além disto, as firmas mais ineficientes cujos escores de eficiência apresentam valores superiores a uma unidade, dão maior 'peso' no efeito parcial da corrupção, levando a um sentido negativo entre as práticas predatórias e o crescimento das firmas. Este padrão de comportamento na amostra é percebido na fronteira total (FDH) além das fronteiras estratificadas, revelando consistências nos resultados. Embora os custos parciais (vinculados aos escores de eficiência) sejam significativamente diferentes, os resultados não apontam divergências na interpretação.

Analisando o módulo do parâmetro dos custos parciais da corrupção, observa-se uma tendência de queda que é alavancada à medida em que as firmas de maior performance na eficiência são incorporadas nos cálculos dos escores parciais (firmas com menor dominância na amostra). No tocante ao poder de explicação do modelo, o $\mathrm{R}^{2}$ apresentou-se relativamente baixo em todos os quantis, embora os efeitos da corrupção ponderados pela eficiência tenham revelado uma forte significância estatística. Os testes de heterocedasticidade levaram à rejeição da hipótese nula, ao nível de $10 \%$ em todos os quantis, de variância constante. Estes resultados revelam indícios de ineficiência nas estimativas, caso o método de bootstrap não fosse devidamente aplicado.

Por fim, as estatísticas de Wald revelaram significância global nos modelos estimados em todo os quantis (significativo ao nível de $1 \%$ ). Estes resultados são coerentes com as significâncias parciais das estimativas dos regressores, rejeitando a hipótese nula de todos os coeficientes serem conjuntamente iguais a zero.

A Tabela 9 apresenta os resultados da equação estimada ME.1 incluindo agora todos os efeitos fixos (país e setor). As estimativas de 
impacto dos investimentos em $\mathrm{P} \& \mathrm{D}$ no crescimento das vendas oscilaram em um intervalo maior em relação aos resultados da Tabela 8, com coeficientes no intervalo de 1,59\% a 1,73\% (parâmetros significativos ao nível de 1\%). As diferenças de intervalos mostram a sensibilidade dos parâmetros associada a características particulares entre os setores, que condicionam os resultados desses investimentos. Esta particularidade também foi percebida nas estimativas associadas à variável número de funcionários: $\ln (\mathrm{L})$. O intervalo de possibilidades dos parâmetros consistiu entre $-4,33$ a $-4,56$ (todos significativos ao nível de 1\%). Novamente, os recursos humanos apresentaram impacto negativo com a taxa de crescimento das firmas.

Tabela 9 - Resultados da equação ME.2

\begin{tabular}{|c|c|c|c|c|c|}
\hline \multirow{3}{*}{ VARIÁVEIS } & \multicolumn{5}{|c|}{ Variável dependente: crescimento das vendas (últimos 3 anos) } \\
\hline & (1) & (2) & (3) & (4) & (5) \\
\hline & Quantil 25\% & Quantil $50 \%$ & Quantil 75\% & Quantil 95\% & FDH \\
\hline \multirow[t]{2}{*}{$\ln (\mathrm{R} \& D)$} & $1.586^{\star \star \star}$ & $1.728^{\star \star \star}$ & $1.703^{\star \star *}$ & $1.723^{\star \star \star}$ & $1.653^{\star \star *}$ \\
\hline & $(0.563)$ & $(0.557)$ & $(0.557)$ & $(0.559)$ & $(0.559)$ \\
\hline \multirow[t]{2}{*}{$\ln (L)$} & $-4.406^{\star \star *}$ & $-4.561^{* * *}$ & $-4.454^{\star \star *}$ & $-4.457^{\star \star *}$ & $-4.327^{\star \star \star}$ \\
\hline & $(0.895)$ & $(0.889)$ & $(0.883)$ & $(0.882)$ & $(0.877)$ \\
\hline \multirow[t]{2}{*}{$\mathrm{CC}$} & $116.3^{*}$ & $113.6^{*}$ & $113.5^{\star}$ & $113.1^{*}$ & $112.9^{*}$ \\
\hline & $(59.51)$ & (59.14) & $(59.18)$ & $(59.10)$ & $(59.07)$ \\
\hline \multirow[t]{2}{*}{$\mathrm{CC}^{*} \lambda_{25}(\mathrm{x}, \mathrm{y})$} & $-8.993^{\star * *}$ & & & & \\
\hline & $(2.297)$ & & & & \\
\hline \multirow[t]{2}{*}{$\mathrm{CC}^{\star} \lambda_{50}(\mathrm{x}, \mathrm{y})$} & & $-5.821^{\star \star \star}$ & & & \\
\hline & & $(0.709)$ & & & \\
\hline \multirow[t]{2}{*}{$\mathrm{CC}^{\star} \lambda_{75}(\mathrm{x}, \mathrm{y})$} & & & $-2.515^{\star \star \star}$ & & \\
\hline & & & $(0.468)$ & & \\
\hline \multirow[t]{2}{*}{$\mathrm{CC}^{\star} \lambda_{95}(\mathrm{x}, \mathrm{y})$} & & & & $-1.675^{\star \star \star}$ & \\
\hline & & & & $(0.114)$ & \\
\hline \multirow[t]{2}{*}{$\mathrm{CC}^{\star} \lambda_{100}(\mathrm{x}, \mathrm{y})$} & & & & & $-1.368^{\star \star \star}$ \\
\hline & & & & & $(0.175)$ \\
\hline Amostra & 2,000 & 2,000 & 2,000 & 2,000 & 2,000 \\
\hline $\mathrm{R}^{2}$ & 0.057 & 0.061 & 0.060 & 0.060 & 0.059 \\
\hline Teste Wald $\left(\chi^{2}\right)$ & $121.15^{\star \star \star}$ & $116.16^{\star \star \star}$ & $108.39^{\star * *}$ & $76.46^{\star * *}$ & $82.96^{\star * *}$ \\
\hline Teste Heterocedasticidade & $3.30^{*}$ & $3.16^{*}$ & $3.06^{*}$ & $3.04^{*}$ & $2.99^{\star}$ \\
\hline Ef.Fixos-Pais & SIM & SIM & SIM & SIM & SIM \\
\hline Ef.Fixos-Setor & SIM & SIM & SIM & SIM & SIM \\
\hline
\end{tabular}

Fonte: Elaboração própria.

Nota: Os asteriscos representam os respectivos p-valores; ${ }^{* *} \mathrm{p}<0.01,{ }^{* *} \mathrm{p}<0.05,{ }^{*} \mathrm{p}<0.1$. As estimativas de erro-padrão foram corrigidas a partir da técnica de bootstrap com 400 réplicas. 
Assim como nos resultados da Tabela 8, o efeito da corrupção no crescimento das vendas é estatisticamente influenciado pelo escore de eficiência das firmas. Mesmo nas economias com alta corrupção, firmas eficientes ou mesmo supereficientes as atividades predatórias apresentam uma influência significativa e positiva no crescimento. Este padrão é observado em todos os quantis, com o destaque do custo parcial (parâmetro vinculado ao escore de eficiência) ser notoriamente superior nas firmas eficientes com maior grau de dominância, ou seja, situada nos quantis inferiores.

Este aspecto revela um importante resultado apresentado nesta pesquisa: efeito direto da corrupção depende de dois vetores de influência, (1) um efeito positivo, que captura padrões relativos à hipótese da "graxa na engrenagem" e (2) um efeito negativo e ponderado pelo grau de eficiência, que reflete um efeito oposto e mais próximo à hipótese da "areia na engrenagem". Assim, firmas ineficientes e com alto valor dos seus escores de eficiência $\left(\lambda_{\alpha}(\mathrm{x}, \mathrm{y})>1\right)$ aumentam o 'peso' negativo da corrupção no crescimento das suas vendas, sugerindo que nas economias não-desenvolvidas, onde a presença de tais firmas seja proporcionalmente maior, os custos da corrupção também sejam mais elevados. Isto pode indicar, ou mesmo sugerir, que os custos da corrupção sejam maiores, tanto nas questões estruturais em tais economias quanto no condicionamento estratégico das empresas que passam a aceitar a corrupção como uma 'jornada natural' na execução dos seus projetos de investimentos. Neste caso, o emprego dos recursos em P\&D passa a ser direcionado para as atividades predatórias e, portanto, sinalizando uma relação negativa com o crescimento da firma.

No tocante às firmas eficientes e supereficientes, estas podem apresentar uma relação 'menos' prejudicial aos negócios com a presença da corrupção. Tal fato sugere que as estratégias voltadas para a dinâmica dos negócios sejam mais centradas nos resultados que buscam 'deslocar' a fronteira e, consequentemente, continuarem na liderança tecnológica. Isto não isenta que nas economias desenvolvidas a presença da corrupção seja nula. Ao contrário, a corrupção torna-se tanto presente, porém, com um padrão provavelmente menos estrutural e mais marginal em relação às economias não desenvolvidas. Tal conclusão é bastante elucidada nas contribuições de Acemoglu e Robinson (2012). Conforme os autores, a origem do fracasso está muito relacionada à ausência da capacidade de algumas nações cons- 
truírem arranjos institucionais que condicionam os incentivos e as penalidades necessárias para o alcanço do sucesso econômico. Esta incapacidade pode condicionar incentivos contrários ao desenvolvimento, podendo explicar porque os investimentos em tais economias apresentam custos de oportunidade relativamente maiores em relação às economias desenvolvidas.

Ainda que as fronteiras parciais venham a convergir para a fronteira total (FDH ou quantil 100\%), o impacto negativo nas economias com alta corrupção é significativamente maior nas firmas mais ineficientes, ao contrário das firmas da fronteira (eficientes). Estimando o valor do escore para as firmas com impacto negativo da corrupção, tem-se a derivada parcial da equação ME. 2 tomando valores estritamente negativos: $\frac{\partial g_{i j c}}{\partial C C_{c}} \equiv \hat{\beta}_{1}-\hat{\beta}_{4} \cdot \tilde{\lambda}_{\alpha}(x, y)_{i j c}<0 \leftrightarrow \tilde{\lambda}_{\alpha}(x, y)_{i j c}>\frac{\hat{\beta}_{1}}{\hat{\beta}_{4}} \mathbf{\square}$. Este resultado pode ser aplicado para cada quantil, conforme o Gráfico 1.

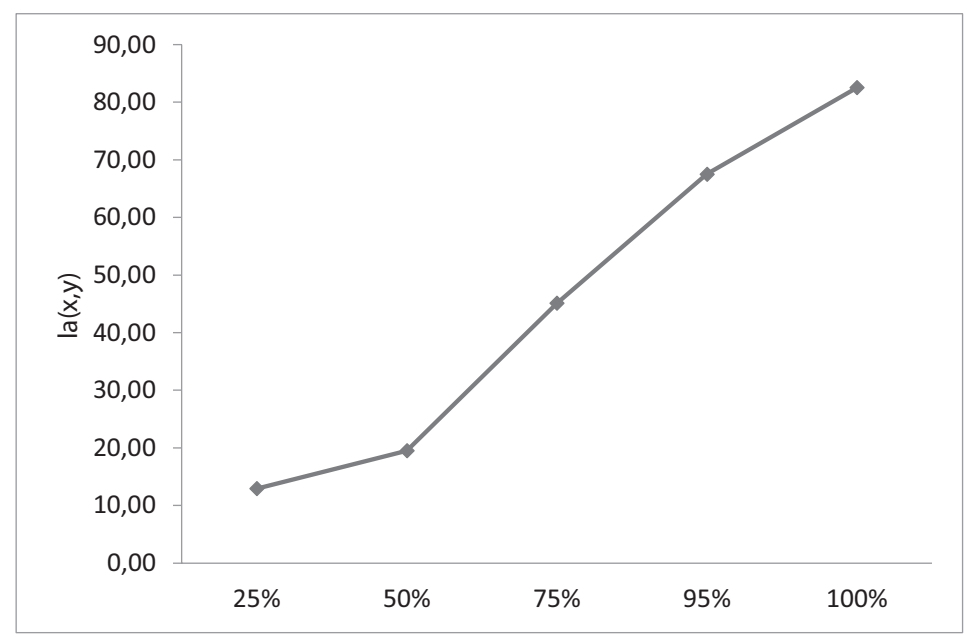

Gráfico 1 - Valores dos escores com efeitos estritamente negativos da corrupção. Fonte: Elaboração própria a partir dos resultados do modelo.

Nota: Os valores estimados consistem nos escores de eficiência por quantil, cujos valores acima implicam em resultados negativos da corrupção no crescimento das vendas.

De acordo com o Gráfico 1, os escores de eficiência que implicam resultados negativos da corrupção no crescimento são mais aderentes à fronteira nos quantis inferiores do que nos quantis superiores. Isto revela que desvios pequenos de eficiência em relação à fronteira 
geram custos negativos da corrupção especialmente nos quantis inferiores, cuja dominância das firmas eficientes é maior em relação às firmas situadas acima do quantil.

No caso em particular, os valores referentes a cada quantil expressam o limite inicial ao qual escores superiores implicam uma relação negativa entre crescimento e corrupção. Considerando o quantil $25 \%$, para firmas que precisam ajustar suas vendas acima de 12,9 milhões de euros, a relação entre os investimentos e o crescimento passa a ser negativa. $\mathrm{O}$ aumento gradativo dos quantis em direção à fronteira final aumenta o tamanho do 'ajuste' que as empresas precisam implementar para moverem em direção à fronteira da eficiência. No limite da fronteira final, o tamanho do ajuste passa a ser notoriamente maior sugerindo que firmas mais ineficientes implicam uma relação negativa entre crescimento e corrupção. Comparando o quantil 95\% com o $100 \%$, a variação no ajuste corresponde a um crescimento de aproximadamente $22 \%$, sendo este influenciado possivelmente pela presença de outliers na amostra (no quantil 95\%, firmas com ajuste acima de 67,52 milhões de euros implicam uma relação negativa entre crescimento e corrupção; contra 82,53 milhões em relação ao quantil 100\%).

$\mathrm{Na}$ medida em que deslocamos as fronteiras parciais em direção aos quantis superiores, o efeito negativo corrupção-ineficiência está mais presente nas firmas mais afastadas da fronteira, em relação aos quantis inferiores.

\subsection{Discussão com recentes estudos}

Os resultados apresentados mostram que nas fronteiras parciais de baixa performance, ou seja, situadas nos quantis inferiores e abaixo da mediana, o custo da corrupção é maior em relação às firmas de alta performance (quantis superiores). Acemoglu, Aghion e Zilibotti (2006) apresentaram evidências mostrando como a trajetória institucional pode conduzir as economias em direção a uma armadilha de não convergência a partir do conceito de distância com a fronteira. Nas economias relativamente mais afastadas da fronteira, as firmas podem implementar estratégias baseadas na adoção de tecnologias provenientes da fronteira, impulsionando rápido crescimento. Políticas direcionadas a esta finalidade restringem importantes 
mecanismos de seleção baseados na restrição de competição no mercado. A persistência destas políticas a longo prazo pode comprometer a trajetória das firmas, direcionando os lucros apropriados, em decorrência da proteção, em estratégias não competitivas, como a corrupção. Tais práticas podem alimentar ainda mais a trajetória institucional, promovendo grandes assimetrias entre as empresas, desfavorecendo especialmente as firmas mais afastadas da fronteira.

Asiedu e Freeman (2009) encontraram resultados significativos da corrupção no crescimento do investimento nas empresas de países em transição. Conforme os autores, "for transition countries, corruption is the most important determinant of investment." (p.200). Entretanto, os resultados apontaram para um impacto não significativo nas firmas da América Latina e da África. As conclusões revelam que a corrupção gera ganhos privados nestes países e que tais ganhos acabam por neutralizar os efeitos negativos sobre os investimentos. Este padrão é possivelmente explicado pelas decisões de investimento que internalizam os efeitos adversos da corrupção, mesmo antes de as empresas iniciarem seus negócios. Este aspecto de 'corrupção estrutural' é mais precisamente percebido em tais economias.

Recentes pesquisas, destacando Wang e You (2012), Wang (2012) e Jiang e Nie (2014), também obtiveram resultados diferenciados da corrupção conforme os níveis de firmas. Conforme Jiang e Nie (2014), a distinção entre firmas de pequeno, médio e grande portes afeta consideravelmente a percepção da corrupção nos resultados operacionais das firmas. As PME's acabam por sofrer maiores consequências com presença da corrupção em relação às firmas de grande porte. Este padrão revela que os custos da corrupção são bastante heterogêneos, especialmente quando incorporamos aspectos particulares das firmas que, em última instância podem ser aproximados pelo grau de eficiência (BATRA, KAUFMANN e STONE, 2003).

\section{Considerações finais}

O presente estudo analisou os efeitos da corrupção nas vendas das empresas em diferentes países, incorporando o grau de eficiência das firmas como fator de ponderação na percepção da corrupção. Adotando uma amostra de 2.000 firmas em 46 países foram cons- 
truídas 5 fronteiras parciais conforme 40 setores, totalizando 200 fronteiras por quantis especificados: $25 \%, 50 \%, 75 \%, 95 \%$ e $100 \%$ ou amostra total.

Os resultados apontam que o efeito da corrupção no crescimento das vendas é estatisticamente influenciado pelo escore de eficiência das firmas. Mesmo nas economias com alta corrupção, firmas eficientes ou mesmo supereficientes, as atividades predatórias apresentam uma influência significativa e positiva no crescimento.

As firmas cujos escores de eficiência que implicam resultados negativos da corrupção no crescimento encontram-se mais aderentes à fronteira nos quantis inferiores em relação aos quantis superiores. Especialmente nos quantis inferiores, pequenos valores nos escores de eficiência geram custos negativos da corrupção. Nestes quantis, a dominância das firmas eficientes é maior em relação às firmas situadas nos quantis superiores, cujos custos da corrupção apresentam ser negativos nas firmas mais ineficientes.

Assim, o efeito 'aproximação com a fronteira' é mais influente para capturar os custos da corrupção nas firmas com maior dominância na amostra. Este aspecto mostra o efeito 'graxa da engrenagem' nas firmas com maior performance, enquanto que nas firmas com baixa performance, pequenos desvios de ineficiência são mais atuantes para determinar o efeito 'areia na engrenagem'.

Esta estratificação na amostra revela que o aspecto médio capturado pelas regressões tradicionais, 'esconde' importantes diferenças que são percebidas em nível de escala e escopo. Neste sentido, os resultados apresentados sugerem uma possível assimetria entre as firmas que é estimulada por falhas institucionais (como a corrupção e a burocracia) e repercutindo em custos relativos, desfavorecendo especialmente as firmas mais ineficientes. Tais resultados corroboram com importantes estudos como Batra, Kaufmann e Stone (2003), Wang e You (2012) e Jiang e Nie (2014).

Por fim, é importante destacar que fatores relativos à endogeneidade das variáveis são uma limitação deste artigo e merecem ser melhor trabalhados em estudos futuros com o intuito de melhor compreender a relação entre corrupção e crescimento das empresas. Trata-se, portanto, de uma janela aberta para futuras pesquisas sobre o tema. 


\section{Referências}

ACEMOGLU, D., \& VERDIER, T. The Choice between Market Failures and Corruption. American Economic Review, 90(1), 194-211, 2000.

ACEMOGLU, D., AGHION, P., \& ZILIBOTTI, F. Distance to Frontier, Selection and Economic Growth. Journal of the European Economic Association, 4(1), 37-74, 2006.

ALEXEEV, M., \& SONG, Y. Corruption and product market competition: An empirical investigation. Journal of Development Economics, 103, 154-166, 2013.

ANDREWS, D., \& BUCHINSKY, M. Evaluation of a Three-Step Method for Choosing the Number of Bootstrap Repetitions. Journal of Econometrics, 103(1-2), 345-386, 2001.

ANOKHIN, S., \& SCHULZE, W. Entrepreneurship, innovation, and corruption. Journal of Business Venturing, 24, 465-476, 2009.

ARAGON, Y., DAOUIA, A., \& THOMAS-AGNAN, C. Nonparametric Frontier Estimation : A Conditional Quantile-based Approach. Econometric Theory, 21(2), 358-389, 2005.

ASIEDU, E., \& FREEMAN, J. The Effect of Corruption on Investment Growth: Evidence from Firms in Latin America, Sub-Saharan Africa, and Transition Countries. Review of Development Economics, 13(2), 200-214, 2009.

BADIN, L., \& DARAIO, C. Explaining Efficiency in Nonparametric Frontier Models: Recent Developments in Statistical Inference. In I. KEILEGOM, \& P. WILSON (Eds.), Exploring Research Frontiers in Contemporary Statistics and Econometrics (pp. 151-175 ). Heidelberg: Springer, 2012.

BADIN, L., DARAIO, C., \& SIMAR, L. How to measure the impact of environmental factors in a nonparametric production model. European Journal of Operational Research, 223, 818-833, 2014.

BAILEY, D. The effects of corruption in a developing nation. Western Political Quarterly, 19(4), 719-732, 1966.

BANERJEE, A., MULlAINATHAN, S., \& HANNA, R. Corruption. Cambridge, MA: National Bureau of Economic Research, NBER Working Papers 17968, 2012.

BATRA, G., KAUFMANN, D., \& STONE, A. Investment Climate Around the World: Voices of the Firms from the World Business Environment Survey. Washington, DC: The World Bank, 2003.

BOGLIACINO, F. Innovation and employment: A firm level analysis with European R\&D Scoreboard data. EconomiA, v. 15, n. 2, p. 141-154, 2014.

BOGLIACINO, F.; CORDONA, S. G. The determinants of R\&D Investment: the role of Cash flow and Capabilities. European Commission's Joint Research Centre (JRC). [S.1.], 2010.

CAMERON, A., \& TRIVEDI, P. Microeconometrics: Methods and Applications. New York: Cambridge University Press, 2005.

CAZALS, C., FLORENS, J., \& SIMAR, L. Nonparametric frontier estimation: a robust approach. Journal of Econometrics, 106, 1-25, 2002.

CHARNES, A., COOPER, W., \& RHODES, E. Measuring the efficiency of decision making units. European Journal of Operational Research, 2, 429-444, 1978.

COMMISSION, E. The 2013 EU Industrial R\&D Investment Scoreboard.Acesso em 15 de jul de 2014, disponível em Publications Office of the European Union: http://ipts.jrc.ec.europa.eu/, 2013.

DAOUIA, A., \& SIMAR, L. Robust Nonparametric Estimators of Monotone Boundaries. Journal of Multivariate Analysis, 96, 311-331, 2005.

DARAIO, C., \& SIMAR, L. Introducing Environmental Variables in Nonparametric Frontier Models: a Probabilistic Approach. Journal of Productivity Analysis, 24(1), 93-121, 2005.

DARAIO, C., \& SIMAR, L. Advanced Robust and Nonparametric Methods in Efficiency Analysis. Springer: New York, NY, 2007. 
DEBREU, G. The coefficient of resource utilization. Econometrica, 19, 273-292, 1951.

DEPRINS, D., SIMAR, L., \& TULKENS, H. Conditional nonparametric frontier models for convex and nonconvex technologies: A unifying approach. Journal of Productivity Analysis, 28, 13-32, 1984.

DREHER, A., \& GASSEBNER, M. Greasing the wheels of entrepreneurship? The impact of regulations and corruption on firm entry. Zurich, Switzerland: Swiss Economic Institute - KOF, Working paper $\mathrm{n}^{\circ}$ 166, 2007.

EFRON, B. Bootstrapping Methods: Another Look at the Jackknife. Annals of Statistics, 7(1), 1-26, 1979.

EFRON, B., \& TIBSHIRANI, R.. An Introduction to the Bootstrap. New York: Chapman \& Hall, 1993.

FARREL, J. The measurement of productive efficiency. Journal of the Royal Statistical Society, A 120 , 253-281, 1957.

FLORENS, J.-P., SIMAR, L., \& KEILEGOM, I. Frontier estimation in nonparametric location-scale models. Journal of Econometrics, 178, 456-470, 2014.

GARCNA-MANJEN, J. V.; ROMERO-MERINO, M. E. Research, development, and firm growth. Empirical evidence from European top R\&D spending firms. Research Policy, v. 41, n. 6, p. 1084- 1092, 2012.

GAVIRIA, A. Assessing the Effects of Corruption and Crime on Firm Performance: Evidence from Latin America. Emerging Markets Review, 3, 245-68, 2002.

GREENE, W. Econometric Analysis (7ª ed.). Boston, MA: Prentice Hall, 2012.

HALLWARD-DRIEMEIER, M., WALLSTERN, S., \& XU, L. The investment climate and the firm: Firm-level evidence from China. Policy Research Working Paper 3003. The World Bank, 2004.

HUNTINGTON, S. Political order in changing societies. New Haven: Yale University Press, 1968.

JIANG, T., \& NIE, H. The stained China miracle: Corruption, regulation, and firm performance. Economics Letters, 123, 366-369, 2014.

KAUFMANN, D., \& WEI, S.-J. Does 'grease money'speed up the wheels of commerce? Cambridge, MA: National Bureau of Economic Research, NBER Working Paper No. 7093, 1999.

KAUFMANN, D., KRAAY, A., \& MASTRUZZI, M. The Worldwide Governance Indicators: Methodology and Analytical Issues. Policy Research Working Paper Series 5430. The World Bank, 2013.

KNEIP, A., SIMAR, L., \& WILSON, P. Asymptotics for DEA Estimators in Nonparametric Frontier Models. Institut de Statistique, UCL. Discussion Paper no. 0317, 2003.

KOENKER, R. Quantile Regression. New York: Cambridge University Press, 2005.

KOENKER, R., \& HALLOCK, K. Quantile Regression. Journal of Economic Perspectives, 15(4), 143-156, 2001.

KOOPMANS, C. An analysis of production as an efficient combination of activities. In T. KOOPMANS (Ed.), Activity Analysis of Production and Allocation (pp. 33-97). New York: John-Wiley and Sons, Inc, 1951.

KUMBHAKAR, S., PARK, B., SIMAR, L., \& TSIONAS, E. Nonparametric stochastic frontiers: A local maximum likelihood approach. Journal of Econometrics, 137(1), 1-27, 2007.

LAMBSDORFF, J. How Corruption Affects Productivity. International Review for Social Sciences, 56(4), 459-476, 2003.

LAMBSDORFF, J. The Institutional Economics of Corruption and Reform: Theory, Evidence, and Policy. New York: Cambridge University Press, 2007.

LEFF, N. Economic development through bureaucratic corruption. In A. HEIDENHEIMER, M. JOHNSTON, \& V. LEVINE, Political corruption: A handbook (pp. 389-403). Oxford, UK: Transaction Books, 1964.

LIVANIS, G.; LAMIN, A. Knowledge, Proximity and R\&D Exodus. Research Policy, v. 45, n. 1, p. 8-26, 2016. 
LUI, F. An equilibrium queuing model of bribery. Journal of Political Economy, 93(4), 760-781, 1985.

MÉNDEZ, F., \& SEPÚLVEDA, F. Corruption, growth and political regimes: Cross country evidence. European Journal of Political Economy, 22, 82-98, 2006.

MÉON, P., \& SEKKAT, K. Does corruption grease or sand the wheels of growth? Public Choice, 122, 69-97, 2005.

MÉON, P., \& WEIL, L. Is corruption an efficient grease? World Development, 38(3), 244-259, 2010.

MOCAN, N. What Determines Corruption? International Evidence from Micro Data. Cambridge, MA: National Bureau of Economic Research, NBER Working Paper No. 10460, 2004.

MONTRESOR, S.; VEZZANI, A. The production function of top R\&D investors: Accounting for size andsector heterogeneity with quantile estimations. Research Policy, v. 44, n. 2, p. 381-393, 2015.

ROSE-ACKERMAN, S. Corruption and Government: Causes, Consequences and Reform. Cambridge, UK: Cambridge University Press, 1999.

SCHUMACHER, I. Political stability, corruption and trust in politicians. Economic Modelling, 31, 359-369, 2013.

SCHWARZ, M., VAN BELLEGEM, S., \& FLORENS, J.-P. Nonparametric Frontier Estimation from Noisy Data. In I. KEILEGOM, \& P. WILSON (Eds.), Exploring Research Frontiers in Contemporary Statistics and Econometrics (pp. 45-64). Heidelberg: Springer, 2012.

SHLEIFER, A., \& VISHNY, R. Corruption. The Quarterly Journal of Economics, 108(3), 599-617, 1993.

SIMAR, L., \& WILSON, P. Sensitivity analysis of efficiency scores: how to bootstrap in nonparametric frontier models. Management Science, 44(1), 49-61, 1998.

SIMAR, L., \& WILSON, P. Statistical Inference in Nonparametric Frontier Models: The State of the Art. The Journal of Productivity Analysis, 13, 49-78, 2000a.

SIMAR, L., \& WILSON, P. A general methodology for bootstrapping in non-parametric frontier models. Journal of Applied Statistics, 27(6), 779-802, 2000b.

SIMAR, L., \& WILSON, P. Estimation and Inference in Nonparametric Frontier Models: Recent Developments and Perspectives. Foundations and Trends in Econometrics, 5(3-4), 183-337, 2013.

SMARZYNSKA, B., \& WEI, S.-J. Corruption and Cross-Border Investment: Firm-Level Evidence. NBER working paper W7969. Cambridge, MA: National Bureau of Economic Research, 2002.

SVENSSON, J. Who must pay bribes and how much? Evidence from a cross section of firms. Quarterly Journal of Economics, 118(1), 207-230, 2003.

TANZI, V. Corruption around the world: causes, scope and cures. IMF Staff Papers, 45, 559-594, 1998.

TREISMAN, D. The causes of corruption: a cross-national study. Journal of Public Economics, 76(3), 399-458, 2000.

WANG, Y., \& YOU, J. Corruption and firm growth: Evidence from China. China Economic Review, 23, 415-433, 2012.

WILSON, P. Asymptotic Properties of Some Non-Parametric Hyperbolic Efficiency Estimators. In I. KEILEGOM, \& P. WILSON (Eds.), Exploring Research Frontiers in Contemporary Statistics and Econometrics (pp. 115-150). Heidelberg: Springer, 2012. 


\section{Apêndice A - Descrição da amostra}

O relatório "The 2013 EU Industrial R\&D Investment Scoreboard" contempla dados econômicos e financeiros das firmas que mais investem em P\&D no mundo, compreendendo um total de 2.000 firmas selecionadas e classificadas pelo investimento realizado em $\mathrm{P} \& \mathrm{D}$. Os dados do relatório são disponibilizados conforme o último balanço disponível pelas firmas, neste caso o ano fiscal de 2012 ou $2012 / 2013$. Os relatórios e contas anuais são documentos de domínio público e coletados pelo departamento 'Industrial Research Monitoring and Analysis (IRMA)' da Comissão Europeia. Os dados coletados são ajustados pelas taxas de câmbio correspondentes nos relatórios anuais tomando como base de referência a data $31 / 12 / 2012$. Além disto, os investimentos realizados pelas empresas correspondem a quantia financiada por cada firma, contemplando ou não o país sede da corporação (investimento total realizado externo e interno).

Esta amostra representa um importante 'retrato' dos esforços em inovação no mundo, uma vez que os dados contemplam as principais firmas investidoras em P\&D e correspondem a uma parcela de $90 \%$ de todo o investimento em pesquisa realizado no mundo, de acordo com informações do próprio relatório. ${ }^{15}$

A seguir são apresentadas as definições e as métricas envolvidas no catálogo de cada variável:

1. Investimentos em Pesquisa e Desenvolvimento (P\&D): representa todo o dispêndio realizado nas atividades de pesquisa, definida como atividades relacionadas a obter novos conhecimentos, sejam eles relacionados diretamente em nível científico ou técnico. Na perspectiva de desenvolvimento, envolve a aplicação de recursos destinados à produção de novos bens ou substancialmente melhorados, aparelhos, produtos, processos, sistemas ou serviços. Os valores dos investimentos são expressos em milhões de euros (€ milhões)]

${ }^{15}$ The Scoreboard is a benchmarking tool which provides reliable up-to-date information on $R \& D$ investment and other economic and financial data, with a unique EU-focus. The 2000 companies listed in this year's Scoreboard account for more than 90\% of worldwide business enterprise expenditure on R\&D (BERD).(COMMISSION, 2013, p. 75) 
2. Investimentos em bens de capital: representa o dispêndio realizado pelas empresas e com foco a adquirir ou atualizar os ativos físicos tais como equipamentos, propriedades, edifícios industriais. $\mathrm{Na}$ conta de 'despesas em capital' é adicionada a conta de ativo (ou seja, capitalizado), aumentando assim a base do ativo. Assim, representa os ativos fixos tangíveis das empresas. Os valores dos investimentos são expressos em milhões de euros ( $€$ milhões).

3. Vendas (líquidas): corresponde à definição contábil das vendas, excluindo os impostos sobre as vendas e participações nas vendas como 'joint ventures' e acionistas. Para os bancos, as vendas são definidas como a renda operacional total mais os rendimentos dos seguros. Com relação a companhias de seguros, as vendas passam a serem definidas como os prêmios brutos emitidos mais quaisquer outros produtos bancários.

4. $\mathrm{N}^{\mathrm{o}}$ de funcionários: consiste na média anual de funcionários empregados ou no resultado demonstrado no final do ano fiscal.

5. Taxa de crescimento (CARG): corresponde a taxa de crescimento anual composta ou 'Compound Annual Growth Rate' (CAGR), obtida pela média geométrica ao longo do período. É calculado a partir da fórmula $g=100 *\left[\left(\frac{A}{B}\right)^{1 / t}-1\right]$, onde $\mathrm{A}=$ variável no ano corrente (2012) e $\mathrm{B}=$ variável no ano base (2011). A variável " $t$ " representa o horizonte do tempo envolvido que, neste caso, consiste em três anos, $t=3$. Tomando $t=1$ tem-se a taxa de crescimento em relação ao período anterior.

\section{Apêndice B - Tabelas cruzadas}

Nesta seção são apresentadas as tabelas cruzando os tipos de firmas conforme os três grupos de setores intensivos em $\mathrm{P} \& \mathrm{D}$. As frequências apresentadas correspondem ao valor absoluto ( $\mathrm{n}^{\mathrm{o}}$ de firmas), à frequência relativa a linha (percentual em relação aos grupos de setores) e a frequência relativa a coluna (percentual em relação aos tipos de firmas). 
Tabela 10 - Tabela de contingência - grau de intensidade X grau de eficiência. Quantil 25\%

\begin{tabular}{ccccccc}
\hline \multirow{2}{*}{ Tipo de setor } & \multicolumn{5}{c}{ Firmas por quantil: 25\% } \\
\cline { 2 - 6 } & Freq. & Eficientes & Ineficientes & Supereficientes & Total \\
\hline \multirow{3}{*}{ Media-intensidade } & Abs. & 49 & 171 & 755 & 975 \\
& Rel. linha (\%) & 5,03 & 17,54 & 77,44 & 100,00 \\
& Rel. coluna (\%) & 51,58 & 45,60 & 49,35 & 48,75 \\
Alta-intensidade & Abs. & 23 & 143 & 647 & 813 \\
& Rel. linha (\%) & 2,83 & 17,59 & 79,58 & 100,00 \\
& Rel. coluna (\%) & 24,21 & 38,13 & 42,29 & 40,65 \\
Baixa-intensidade & Abs. & 23 & 61 & 128 & 212 \\
& Rel. linha (\%) & 10,85 & 28,77 & 60,38 & 100,00 \\
& Rel. coluna (\%) & 24,21 & 16,27 & 8,37 & 10,60 \\
\hline \multirow{4}{*}{ Total } & Abs. & 95 & 375 & 1.530 & 2.000 \\
& Rel. linha (\%) & 4,75 & 18,75 & 76,50 & 100,00 \\
& Rel. coluna (\%) & 100,00 & 100,00 & 100,00 & 100,00 \\
\hline
\end{tabular}

Fonte: Elaboração própria.

Tabela 11 - Tabela de contingência - grau de intensidade X grau de eficiência. Quantil 50\%

\begin{tabular}{cccccc}
\hline \multirow{2}{*}{ Tipo de setor } & \multicolumn{5}{c}{ Firmas por quantil: $\mathbf{5 0 \%}$} \\
\cline { 2 - 6 } & Freq. & Eficientes & Ineficientes & Supereficientes & Total \\
\hline \multirow{3}{*}{ Media-intensidade } & Abs. & 52 & 189 & 734 & 975 \\
& Rel. linha (\%) & 5,33 & 19,38 & 75,28 & 100,00 \\
& Rel. coluna (\%) & 46,02 & 45,54 & 49,86 & 48,75 \\
& Abs. & 34 & 163 & 616 & 813 \\
\multirow{3}{*}{ Alta-intensidade } & Rel. linha (\%) & 4,18 & 20,05 & 75,77 & 100,00 \\
& Rel. coluna (\%) & 30,09 & 39,28 & 41,85 & 40,65 \\
& Abs. & 27 & 63 & 122 & 212 \\
& Rel. linha (\%) & 12,74 & 29,72 & 57,55 & 100,00 \\
& Rel. coluna (\%) & 23,89 & 15,18 & 8,29 & 10,60 \\
\hline \multirow{7}{*}{ Total } & Abs. & 113 & 415 & 1.472 & $\mathbf{2 . 0 0 0}$ \\
& Rel. linha (\%) & $\mathbf{5 , 6 5}$ & $\mathbf{2 0 , 7 5}$ & $\mathbf{7 3 , 6 0}$ & 100,00 \\
& Rel. coluna (\%) & 100,00 & 100,00 & 100,00 & 100,00 \\
\hline
\end{tabular}

Fonte: Elaboração própria. 
Tabela 12 - Tabela de contingência - grau de intensidade X grau de eficiência. Quantil 75\%

\begin{tabular}{cccccc}
\hline \multirow{2}{*}{ Tipo de setor } & \multicolumn{5}{c}{ Firmas por quantil: $75 \%$} \\
\cline { 2 - 6 } & Freq. & Eficientes & Ineficientes & Supereficientes & Total \\
\hline \multirow{3}{*}{ Media-intensidade } & Abs. & 127 & 241 & 607 & 975 \\
& Rel. linha (\%) & 13,03 & 24,72 & 62,26 & 100,00 \\
& Rel. coluna (\%) & 53,36 & 44,80 & 49,59 & 48,75 \\
\multirow{3}{*}{ Alta-intensidade } & Abs. & 56 & 222 & 535 & 813 \\
& Rel. linha (\%) & 6,89 & 27,31 & 65,81 & 100,00 \\
& Rel. coluna (\%) & 23,53 & 41,26 & 43,71 & 40,65 \\
\multirow{3}{*}{ Baixa-intensidade } & Abs. & 55 & 75 & 82 & 212 \\
& Rel. linha (\%) & 25,94 & 35,38 & 38,68 & 100,00 \\
& Rel. coluna (\%) & 23,11 & 13,94 & 6,70 & 10,60 \\
\hline \multirow{2}{*}{ Total } & Abs. & 238 & 538 & 1.224 & 2.000 \\
& Rel. linha (\%) & 11,90 & 26,90 & 61,20 & 100,00 \\
& Rel. coluna (\%) & 100,00 & 100,00 & 100,00 & 100,00 \\
\hline
\end{tabular}

Fonte: Elaboração própria.

Tabela 13 - Tabela de contingência - grau de intensidade X grau de eficiência. Quantil 95\%

\begin{tabular}{cc|cccc}
\hline \multirow{2}{*}{ Tipo de setor } & \multicolumn{5}{c}{ Firmas por quantil: 95\% } \\
\cline { 2 - 6 } & Freq. & Eficientes & Ineficientes & Supereficientes & Total \\
\hline \multirow{2}{*}{$\begin{array}{c}\text { Media-intensi- } \\
\text { dade }\end{array}$} & Abs. & 291 & 447 & 237 & 975 \\
& Rel. linha (\%) & 29,85 & 45,85 & 24,31 & 100,00 \\
& Rel. coluna (\%) & 57,85 & 44,97 & 47,12 & 48,75 \\
Alta-intensidade & Abs. & 124 & 438 & 251 & 813 \\
& Rel. linha (\%) & 15,25 & 53,87 & 30,87 & 100,00 \\
& Rel. coluna (\%) & 24,65 & 44,06 & 49,90 & 40,65 \\
& Abs. & 88 & 109 & 15 & 212 \\
Baixa-intensidade & Rel. linha (\%) & 41,51 & 51,42 & 7,08 & 100,00 \\
& Rel. coluna (\%) & 17,50 & 10,97 & 2,98 & 10,60 \\
\hline \multirow{2}{*}{ Total } & Abs. & $\mathbf{5 0 3}$ & $\mathbf{9 9 4}$ & $\mathbf{5 0 3}$ & $\mathbf{2}, 000$ \\
& Rel. linha (\%) & $\mathbf{2 5 , 1 5}$ & $\mathbf{4 9 , 7 0}$ & $\mathbf{2 5 , 1 5}$ & 100,00 \\
& Rel. coluna (\%) & 100,00 & 100,00 & 100,00 & 100,00 \\
\hline
\end{tabular}

Fonte: Elaboração própria. 
Tabela 14 - Tabela de contingência - grau de intensidade X grau de eficiência. Quantil 100\%

\begin{tabular}{cc|cccc}
\hline \multirow{2}{*}{ Tipo de setor } & \multicolumn{5}{c}{ Firmas por quantil: 100\% } \\
\cline { 2 - 6 } & Freq. & Eficientes & Ineficientes & Supereficientes & Total \\
\cline { 2 - 6 } Media-intensidade & Abs. & 379 & 596 & - & 975 \\
& Rel. linha (\%) & 38,87 & 61,13 & & 100,00 \\
& Rel. coluna (\%) & 57,77 & 44,35 & 48,75 \\
& Abs. & 177 & 636 & 813 \\
Alta-intensidade & Rel. linha (\%) & 21,77 & 78,23 & 100,00 \\
& Rel. coluna (\%) & 26,98 & 47,32 & 40,65 \\
& Abs. & 100 & 112 & 212 \\
Baixa-intensidade & Rel. linha (\%) & 47,17 & 52,83 & 100,00 \\
& Rel. coluna (\%) & 15,24 & 8,33 & 10,60 \\
\hline \multirow{2}{*}{ Total } & Abs. & 656 & 1.344 & - & 2.000 \\
& Rel. linha (\%) & 32,80 & 67,20 & 100,00 \\
& Rel. coluna (\%) & 100,00 & 100,00 & 100,00 \\
\hline
\end{tabular}

Fonte: Elaboração própria. 